Pacific Journal of Mathematics

FUNCTIONAL GILBERTIAN SUMS 


\title{
FUNCTIONAL HILBERTIAN SUMS
}

\author{
HASKell Rosenthal
}

A real Banach space is called a Functional Hilbertian Sum (FHS) if it is isometric to the direct sum of Hilbert spaces of dimension at least two via a one-unconditional basis. Various isometric permanence properties of Functional Hilbertian Sums are proved. Many of these results are the real analogues of (and also imply) known theorems concerning complex Banach spaces with one-unconditional (or "hyperorthogonal") bases. For example, it is proved that a space is FHS if and only if it equals the closed linear span of the ranges of its rank-two skew-Hermitian operators. The complex analogue due to Kalton and Wood is as follows: a complex Banach space has a one-unconditional basis provided it equals the closed linear span of the ranges of its rank-one skew-Hermitian operators. The isometries and skew-Hermitian operators on FHS spaces are completely determined and FHS spaces are isometrically classified. Skew-Hermitian operators on general real spaces with a one-unconditional basis are also completely determined, using FHS spaces in an essential manner. Various complementation results are established, insuring that under certain circumstances, one-complemented subspaces of spaces with one-unconditional bases are FHS spaces. One of these yields the real analogue of (and also implies) the theorem of Kalton and Wood that the family of complex Banach spaces with one-unconditional bases is closed under contractive projections. In the course of this investigation, several isometric invariants for real Banach spaces are introduced. Many of these are natural analogues of known invariants for complex spaces and include orthogonal projections, well-embedded spaces, Hilbert components and B. Lie algebras.

TABLE OF CONTENTS

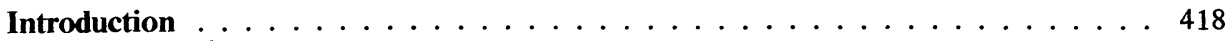

Section 1. Preliminaries . . . . . . . . . . . . . . . . . . 421

B. Lie algebras and compatible norms . . . . . . . . . . . . . . . 421

Orthogonal decompositions . . . . . . . . . . . . . . . . 425

Well-embedded Hilbert spaces and Hilbert components. . . . . . . . . . . . . . 430

Section 2. Characterizations of well-embedded Hilbert subspaces and the struc-

ture of Hilbert components . . . . . . . . . . . . . . . . . . . . . . . 432

Lie algebra characterizations of rotation spaces and well-embedded Euclidean

spaces . . . . . . . . . . . . . . . . . . . . . 434

Orthogonality of Hilbert components . . . . . . . . . . . . . 441

Lie-classification of spaces of low dimension. . . . . . . . . . . . . . 443

Section 3. The isometric structure of FHS . . . . . . . . . . . . . . . . . 445

The Functional Hilbertian part of a Banach space and the structure

of spaces with a one-unconditional basis. . . . . . . . . . . . . 446

Detailed structure of FHS spaces and their isometry groups. . . . . . . . . 456

Complementation results. . . . . . . . . . . . . . . 460

References . . . . . . . . . . . . . . . . . . . 466 
0. Introduction. Let $\Gamma$ be a nonempty set and $\left(X_{\alpha}\right)_{\alpha \in \Gamma}$ a family of nonzero Banach spaces. A Banach space $B$ is said to be a functional unconditional sum of the $X_{\alpha}$ 's if there exists a normalized one-unconditional basis $\underline{u}=\left(u_{\alpha}\right)_{\alpha \in \Gamma}$ for some Banach space $U$ so that $B$ is (linearly isometric to) $\left(\Sigma_{\Gamma} \oplus X_{\alpha}\right)_{\underline{u}}$; the latter refers to the Banach space consisting of all $x=\left(x_{\alpha}\right)_{\alpha \in \Gamma}$ in $\Pi_{\alpha \in \Gamma} X_{\alpha}$ with $\sum_{\alpha \in \Gamma}\left\|x_{\alpha}\right\| u_{\alpha}$ in $U$ under the norm $\|x\|=\left\|\sum_{\alpha \in \Gamma}\right\| x_{\alpha}\left\|u_{\alpha}\right\|_{U}$. In case $B$ is real and each $X_{\alpha}$ is a real Hilbert space of dimension at least equal to two, we call $B$ a Functional Hilbertian Sum and denote the class of such spaces by FHS. We shall abuse English at times by using the terminology " $B$ is FHS" to mean that $B$ belongs to FHS. (For complex spaces, one-unconditional bases are called "hyperorthogonal bases" by some authors and, if normalized, "orthonormal systems" by others. Also note that a separable Banach space has a one-unconditional basis if and only if it is isometric to a Banach sequence space with an absolute norm.) Throughout, " $B$ " shall always refer to some real or complex nonzero Banach space. The Lie algebra of $B$, denoted by $\mathfrak{A}(B)$, refers to the set of all operators $T$ on $B$ with $\mathscr{R e} f(T b)=0$ for all $b \in B$ and $f \in B^{*}$ with $f(b)=\|f\|\|b\|$. We refer to the members of $\mathfrak{A}(B)$ as skew-Hermitian operators. "Operator" means "bounded linear map" and "subspace" means "closed linear submanifold."

If $B$ is complex, we may define $T \in \mathscr{L}(B)$ to be Hermitian if $i T$ is skew-Hermitian. Many of our results are known for complex Banach spaces and follow from the rather well developed theory of Hermitian operators. Our focus is on the structure of real Banach spaces; hence we emphasize the Lie algebra of skew-Hermitian operators. In section one, we revew various equivalences and properties of $\mathfrak{A}(B)$, including the fact that this is indeed the Lie algebra of the group of isometries of $B$. The Lie algebra of a Banach space was first formally introduced in [10], and several basic properties were established there. (For basic results about Lie groups and Lie algebras, see [5] or [15].)

We prove the following result in section three below:

THEOREM 1. Let $B$ be real. The following are equivalent:

(a) $B$ is a Functional Hilbertian Sum.

(b) $B$ equals the closed linear span of its rank-two skew-Hermitian operators.

This result is the real analogue of a theorem of Kalton and Wood [6] concerning complex Banach spaces. Indeed, they show that a complex 
Banach space has a one-unconditional basis if (and only if) it is the closed linear span of its rank-one skew-Hermitian operators. The relationship between these two results is discussed in section three; note that a real Banach space $X$ equals $B_{\mathrm{R}}$ for some complex space $B$ with a one-unconditional basis if and only $X$ is a functional unconditional sum of even-dimensional Hilbert spaces. ( $B_{\mathbf{R}}$ denotes $B$ regarded as a real Banach space.)

In section three, we completely classify the spaces in FHS up to isometry and give a number of their isometric properties. To formulate this classification, we present the following concept: Let $U$ be a real

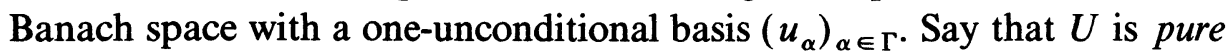
if $U$ has no rank-two skew-Hermitian operators; otherwise say that $U$ is impure. We show in Corollary 3.4 that $U$ is impure if and only if there exist $\alpha \neq \beta$ so that $\left[u_{\alpha}, u_{\beta}\right]$ is the range of a skew-Hermitian operator $T$ on $U$; the kernel of $T$ necessarily equals $\left[u_{j}\right]_{\Gamma \sim\{\alpha, \beta\}}$. We also prove in Theorem 3.11 that if $U$ is pure, then $U$ has no nonzero skew-Hermitian operators. (Throughout, $[A]$ denotes the closed linear span of the set $A$; also if $A=\left\{a_{\alpha}: \alpha \in \Gamma\right\}$, we let $\left[a_{\alpha}\right]_{\Gamma}=[A]=\left[a_{\alpha}\right]$ if $\Gamma$ is understood.) We may formulate the classification for separable spaces as follows: (for this result only, let $\Gamma=\{1,2, \ldots, n\}$ for some positive integer $n$ or $\Gamma=N=\{1,2, \ldots\})$.

THEOREM 2. Let B be a separable FHS. There exists a pure separable $U$ with a normalized one-unconditional basis $\underline{u}=\left(u_{j}\right)$ and separable non-onedimensional Hilbert spaces $\left(H_{j}\right)$ so that $B$ is (isometric to) $\left(\sum_{\Gamma} \oplus H_{j}\right)_{\underline{u}}$. The basis $\left(u_{j}\right)$ and the $H_{j}$ 's are isometrically uniquely determined (up to permutation). That is, if $B$ is also isometric to $\left(\Sigma_{\Gamma^{\prime}} \oplus H_{j}^{\prime}\right)_{v}$ with $\left(v_{j}\right)$ a normalized one-unconditional basis for $V$, then $\Gamma=\Gamma^{\prime}$ and there exists $a$ one-one onto map $\sigma: \Gamma \rightarrow \Gamma$ with $\left(v_{\sigma(j)}\right)$ isometrically equivalent to $\left(u_{j}\right)$ and $H_{\sigma(j)}^{\prime}$ isometric to $H_{j}$ for all $j$.

Evidently this result yields in particular that FHS spaces have isometrically unique unconditional bases, up to permutation. We also show in section three that every one-unconditional basis splits into two pieces, such that one spans an FHS space which "generates" the Lie algebra of the space. That is, given $\left(u_{\alpha}\right)_{\alpha \in \Gamma}$ a one-unconditional basis for some real $B$, there exist unique disjoint subsets $\Gamma_{1}$ and $\Gamma_{2}$ of $\Gamma$ so that $\mathrm{FH}(B)=\left[u_{\alpha}\right]_{\alpha \in \Gamma_{1}}$ is FHS, and such that an operator $T$ on $B$ is skew-Hermitian if and only if $T \mid \mathrm{FH}(B)$ is skew-Hermitian and $T \mid \mathcal{O}(\mathrm{FH}(B))=0$ where $\mathcal{O}(\mathrm{FH}(B))=$ $\left[u_{\alpha}\right]_{\alpha \in \Gamma_{2}}$. Moreover every skew-Hermitian operator on $\mathrm{FH}(B)$ extends to 
one on $B$. We also give a complete description of the skew-Hermitian operators and the group of isometries of an arbitrary FHS space. In particular, we thus obtain a detailed description of the component of the identity of the group of isometries of a finite-dimensional Banach space with a one-unconditional basis.

It is evident that if $B$ is complex with a one-unconditional basis, then $B_{\mathbf{R}}$ is a functional unconditional sum of two-dimensional Hilbert spaces, where $B_{\mathbf{R}}$ denotes $B$ regarded as a real Banach space. Our results then yield the isometric classification of such complex spaces, as well as the characterizations of their groups of isometries and Lie algebras. For example, Theorem 2 holds as stated for arbitrary separable complex spaces $B$ with a one-unconditional basis, except that one simply deletes the requirement that the Hilbert spaces $\left(H_{j}\right)$ be non-one-dimensional. The characterizations of the isometries and Lie algebras of complex Banach spaces with one-unconditional basis are known. They were established by Schneider and Turner [11] for finite-dimensional complex spaces, and by Fleming and Jameson in [3] and [4], for the separable infinite-dimensional ones. (The generalization to spaces of arbitrary cardinality presents no essential difficulties, and we present this in section three also.) Suppose $B$ is complex with a one-unconditional basis $\left(u_{\alpha}\right)_{\Gamma}$ and let $U$ denote the real closed linear span of the $u_{\alpha}$ 's. An equivalence relation on the basis $\left(u_{\alpha}\right)$ was introduced in [11], for finite-dimensional spaces. It turns out that $u_{\alpha}$ is equivalent to $u_{\beta}$ in $B$ if and only if $\left[u_{\alpha}, u_{\beta}\right]$ is the range of a rank-two skew-Hermitian operator on $U$. We also note that functional unconditional decompositions of complex Hilbert spaces are introduced in [3] and termed there " $H$-decompositions." We mention these results for their historical importance and to provide a guide for those familiar with them. Our presentation below is however self-contained and formally independent of these earlier results.

One of the open problems which motivated our work is the following one: Suppose $B$ has an unconditional basis and $X$ is complemented in $B$. Does $X$ have an unconditional basis? It is easily seen that one may assume $B$ has a one-unconditional basis. For real spaces, the problem is open even if it is assumed $X$ is one-complemented; i.e., the range of a contractive projection. It follows from the results in [6] (see [9]) that if $X$ is one-complemented in such a $B$ with $B$ complex, then $X$ indeed has a one-unconditional basis (over the complex numbers). We recover this result here by proving a suitable analogue for FHS spaces $B$ (see Theorem 3.15). We also prove that a real Banach space $X$ has a one-unconditional basis if (and only if) $X$ is (isometric to) an orthogonally complemented 
subspace of an FHS space. (The concept of orthogonal complementation is developed in section two; this has been previously studied for complex spaces, but apparently not for real ones.)

We also complete here some investigations begun in [10]. A Banach space is called Euclidean if it is isometric to a Hilbert space. A real Banach space $B$ is called a rotation space if $\operatorname{dim} B \geq 3$ and $B$ is a functional unconditional sum of a Hilbert space and a one-dimensional space.

THEOREM 3. Let $B$ be real $n$-dimensional with $n \geq 2$.

(a) $B$ is Euclidean if $\operatorname{dim} \mathfrak{U}(B)>(n-1)(n-2) / 2$ (and then $\operatorname{dim} \mathfrak{A}(B)=n(n-1) / 2)$.

(b) $B$ is a non-Euclidean rotation space if and only if $n \geq 3$ and $\operatorname{dim} \mathfrak{A}(B)=(n-1)(n-2) / 2$.

Theorem 3(a) as well as the easy "only if" assertion in 3(b) were established in [10]. We give a self-contained proof of Theorem 3 in section two, as well as completing the classification of the Lie algebras of four-dimensional spaces stated in [10].

The remainder of this work is divided in three sections. In section one we introduce all the relevant concepts and recall results from [10]. We also prove simple things about the concepts, which include orthogonality in real Banach spaces, compatible norms, well-embedded Hilbert spaces, and Hilbert components. (The latter notion was introduced for complex spaces in [6] by Kalton and Wood; our development is substantially different.) Section two contains deeper results characterizing well-embedded Hilbert spaces and Hilbert components. In particular, we establish the following fundamental result in Theorem 2.3: distinct Hilbert components are orthogonal provided one of them is at least two-dimensional. Section three is devoted to establishing various isometric properties of FHS spaces. For example, the general version of Theorem 2 is given as Theorem 3.7; an explicit description of the isometric classification of FHS spaces is given in Theorem 3.14. The Lie algebra of an arbitrary space with one-unconditional basis is described in Theorem 3.8 and Corollary 3.11, while the group of isometries of an FHS space is given by Theorem 3.12 .

\section{Preliminaries.}

B. Lie algebras and compatible norms.

We begin by recalling some facts concerning the Lie algebra of a general space $B$. Recall, $\mathfrak{A}(B)$ denotes the (real linear) space of skewHermitian operators on $B$; we let $\mathscr{I}(B)$ denote the set of linear surjective 
isometries of $B . \mathscr{I}(B)$ is of course a topological group under the normoperator topology; if $B$ is finite-dimensional, $\mathscr{I}(B)$ is a Lie group. $\mathscr{L}(B)$ denotes the space of operators on $B$.

THEOREM 1.1. Let $T$ be an operator on $B$. The following are equivalent:

(a) $T$ is skew-Hermitian.

(b) $\left\|e^{x T}\right\| \leq 1$ for all real $x$.

(c) $e^{x T}$ is in $\mathscr{I}(B)$ for all real $x$.

(d) $T$ belongs to the tangent space of $\mathscr{I}(B)$ at $I$.

(e) $\lim _{x \rightarrow 0}(\|I+x T\|-1) / x=0$.

For a proof, see Theorem 1.4 of [10]. If $B$ is finite-dimensional, notice that (d) shows that $\mathfrak{A}(B)$ is precisely the Lie algebra of the Lie group $\mathscr{I}(B)$. We recall also the following result ("projection" means "idempotent operator"):

Proposition 1.2. Let $S$ and $T$ be skew-Hermitian operators on $B$ and $U$ in $\mathscr{I}(B)$. Let $X$ be a subspace of $B$ and $P: B \rightarrow X$ a norm-one projection from $B$ onto $X$.

(a) $U^{-1} T U$ is skew-Hermitian.

(b) $\mathfrak{A}(B)$ is a weak-operator-closed real linear space.

(c) TS - ST is skew-Hermitian.

(d) $P T \mid X$ is in $\mathfrak{A}(X)$.

This is Proposition 1.5 of [10]. In general, for an operator $T$ on $B$ and $P$ as above, the operator $P T \mid X$ is called the compression of $T$ to $X$ (via $P$ ). Thus (d) asserts that if $T$ is skew-Hermitian on $B$, the compression of $T$ to $X$ is skew-Hermitian on $X$. For operators $S$ and $T$ on $B$, we define the Lie bracket of $S$ and $T,[S, T]$, by $[S, T]=S T-T S$.. Thus (c) shows $\mathfrak{A}(B)$ is closed under Lie-brackets.

We recall now the concept of a compatible norm. Let $B$ with norm $\|\cdot\|$ be isomorphic to Hilbert space and $\|\cdot\|_{H}$ be an equivalent norm on B. $\|\cdot\|$ and $\|\cdot\|_{H}$ are called compatible $\left(\|\cdot\|_{H}\right.$ is a compatible norm on $B)$ if

(a) $H$ is Euclidean (where $H=\left(B,\|\cdot\|_{H}\right)$ ) and

(b) $\mathscr{I}(B) \subset \mathscr{I}(H)$.

Note that if $B$ is already Euclidean, then for any $x$ and $y$ in $B$ with $\|x\|=\|y\|$ there is a $T \in \mathscr{I}(B)$ with $T x=T y$. Hence if $\|\cdot\|$ and $\|\cdot\|_{H}$ are compatible, $\|\cdot\|_{H}$ is a nonzero multiple of $\|\cdot\|$ (and so $\|\cdot\|_{H}$ and $\|\cdot\|$ are compatible since then $\mathscr{I}(B)=\mathscr{I}(H)$ ). 
We let $E_{n}$ denote the standard real Euclidean space $\mathbf{R}^{n}$ endowed with the norm $\|x\|_{E_{n}}=\left(\sum_{i=1}^{n} x_{i}^{2}\right)^{1 / 2}$ for $x=\left(x_{1}, \ldots, x_{n}\right)$ in $\mathbf{R}^{n}$. We identify $\mathscr{L}\left(E_{n}\right)$ with all $n \times n$ matrices of real numbers. Thus $\mathscr{I}\left(E_{n}\right)=\mathcal{O}_{n}$, the real orthogonal group. We also let $S O_{n}$ denote the special orthogonal group, that is, the members of $\mathcal{O}_{n}$ of determinant one. $s \mathfrak{D}_{n}$ denotes the family of all $n \times n$ skew-symmetric real matrices; of course $s \mathfrak{0}_{n}=\mathfrak{A}\left(E_{n}\right)$, the Lie algebra of $S \mathcal{O}_{n}$. Subsets $A$ and $B$ of $\mathbf{R}^{n}$ are called orthogonally equivalent if there is a $U \in \mathcal{O}_{n}$ with $U^{*} A U=B$. The following result is established in Propositions 3.3 and 3.4 of [10] (most of the assertions follow easily from 1.1 and 1.2).

Proposition 1.3. Let $B$ be given.

(a) Suppose $\|\cdot\|_{H}$ is a compatible norm on $B$ and $\langle$,$\rangle is an inner$ product on $B$ inducing $\|\cdot\|_{H}$. Then $\mathfrak{A}(B) \subset \mathfrak{A}(H) ; T \in \mathfrak{A}(H)$ if and only if $T^{*}=-T$ (where " $T^{*}$ " denotes the Hilbert space adjoint of $T$.)

(b) If $B$ is finite-dimensional, $B$ has a compatible norm.

(c) Suppose $\|\cdot\|_{1}$ and $\|\cdot\|_{2}$ are norms on $\mathbf{R}^{n}$, each compatible with $\|\cdot\|_{E_{n}}$ such that $B_{1}$ and $B_{2}$ are isometric, where $B_{i}=\left(\mathbf{R}^{n},\|\cdot\|_{i}\right)$ for $i=1$, 2. Then $\mathfrak{A}\left(B_{1}\right)$ and $\mathfrak{A}\left(B_{2}\right)$ are orthogonally equivalent.

We now introduce some concepts and recall Robbin's elegant characterization of the Lie algebra of a finite-dimensional Banach space.

Let $B$ be given and $\mathfrak{A}$ a subset of $\mathscr{L}(B) . \mathfrak{A}$ is said to be saturated if wherever $T \in \mathscr{L}(B)$ is such that $T x \in \mathfrak{A} x$ for all $x \in B$, then $T \in \mathfrak{A}$. $(\mathfrak{A} x=\{A x: A \in \mathfrak{A}\}$.) $\mathfrak{A}$ is said to be a Lie algebra if $\mathfrak{A}$ is a real linear space closed under Lie brackets; i.e., $[S, T]$ is in $\mathscr{U}$ whenever $S$ and $T$ are in $\mathfrak{U} . \mathfrak{A}$ is said to be a $B$. Lie algebra if there is an equivalent norm $\|\cdot\|^{\prime}$ on $B$ so that $\mathfrak{A}=\mathfrak{A}\left(B^{\prime}\right)$ where $B^{\prime}=\left(B,\|\cdot\|^{\prime}\right)$. ("B." stands for"Banach." We don't explictly use the terminology "Banach Lie algebra" since this is already commonly employed in a more general context.) It is evident that any $B$. Lie algebra is a saturated Lie algebra. The following result yields the appropriate converse for finite-dimensional spaces.

THEOREM 1.4. Let $n$ be a positive integer, $B$ an $n$-dimensional real space and $\mathfrak{A}$ a subset of $\mathscr{L}(B)$. Then the following are equivalent:

(a) $\mathfrak{A}$ is a B. Lie algebra.

(b) $\mathfrak{U}$ is a saturated Lie algebra such that $\left\{e^{A}: A \in \mathfrak{U}\right\}$ is a compact group.

(c) $\mathfrak{A}$ is saturated and the Lie algebra of a compact group of automorphisms of $B$. 
Moreover if $B=\mathbf{R}^{n}$ and $\mathfrak{A}$ is a $B$. Lie algebra with $\mathfrak{A} \subset s \mathfrak{o}_{n}$, there is a norm $\|\cdot\|^{\prime}$ on $\mathbf{R}^{n}$ with $\|\cdot\|^{\prime}$ and $\|\cdot\|_{E_{n}}$ compatible so that $\mathfrak{A}=\mathfrak{A}\left(B^{\prime}\right)$ with $B^{\prime}=\left(\mathbf{R}^{n},\|\cdot\|^{\prime}\right)$.

Notice that if $G$ is a compact group of automorphisms of $B$, then defining $\|b\|^{\prime}=\sup _{g \in G}\|g b\|, G$ is a compact group of isometries of $B^{\prime}=\left(B,\|\cdot\|^{\prime}\right)$; since there is then a compatible norm on $B^{\prime}$, we have that $G$ is isomorphic to a closed subgroup of $\mathcal{O}_{n}$ and is hence a Lie group; we may define the Lie algebra of $G$ to be the tangent space to $G$ at $I$. We also observe that Robbin's result easily generalizes to the complex case as noted in [8]: Suppose $B$ is a complex $n$-dimensional space and $\mathfrak{A} \subset \mathscr{L}(B)$. Then $\mathfrak{A}$ is a $B$. Lie algebra if and only if $\mathfrak{A}$ is a $B$. Lie algebra of $B_{\mathbf{R}}$ such that $i I \in \mathfrak{A}$.

The equivalences (a) $\Leftrightarrow$ (c) are due to Robbin [8] ((a) $\Rightarrow$ (b) follows from rather deep results in the classical theory of Lie groups). The final statement is somewhat of an improvement of the result stated as Theorem 3.9 in [10], so we sketch its proof here.

We first recall the following elementary fact: If $R$ is a positive definite matrix and $U$ and $V$ are orthogonal matrices with $R^{-1} U R=V$, then $[U, R]=0$ so $U=V$. (For a proof, see the argument for Theorem 3.4(b) in [10].) It then follows immediately that the same is true for skew-symmetric matrices; that is,

$$
\begin{aligned}
& R^{-1} S R=T \text { and } S, T \in S \mathcal{O}_{n}, \\
& R \text { positive definite implies }[S, R]=0 \text { so } S=T .
\end{aligned}
$$

Indeed, simply apply the result to $U_{x}=e^{x S}$ and $V_{x}=e^{x T}$ for all real $x$, then differentiate and set $x=0$.

Now suppose $\mathfrak{A} \subset s \mathcal{O}_{n}$ is a $B$. Lie algebra and let $\|\cdot\|$ be a norm on $\mathbf{R}^{n}$ with $\mathfrak{A}=\mathfrak{A}(B)$ with $B=\left(\mathbf{R}^{n},\|\cdot\|\right)$. Choose $\|\cdot\|^{\prime}$ a norm on $\mathbf{R}^{n}$ with $\|\cdot\|_{E_{n}}$ and $\|\cdot\|^{\prime}$ compatible and $B^{\prime}$ isometric to $B$ where $B^{\prime}=\left(\mathbf{R}^{n},\|\cdot\|^{\prime}\right)$. Let $T: B \rightarrow B^{\prime}$ be a linear isometry. Then evidently

$$
\mathfrak{U}(B)=T^{-1} \mathfrak{U}\left(B^{\prime}\right) T .
$$

Now choose $U$ orthogonal and $R$ positive definite with $T=U R$. Then (1) and (2) yield that

$$
\mathfrak{A}(B)=U^{*} \mathfrak{A}\left(B^{\prime}\right) U
$$

Finally define $\|\cdot\|^{\prime \prime}$ on $\mathbf{R}^{n}$ by $\|x\|^{\prime \prime}=\|U x\|^{\prime}$ for all $x \in \mathbf{R}^{n}$ and set $B^{\prime \prime}=\left(\mathbf{R}^{n},\|\cdot\|^{\prime \prime}\right)$. Then $\|\cdot\|^{\prime \prime}$ and $\|\cdot\|_{E_{n}}$ are compatible and (3) yields that $\mathfrak{U}=\mathfrak{A}\left(\boldsymbol{B}^{\prime \prime}\right)$, completing the proof. 
Let us say that Banach spaces $B$ and $B^{\prime}$ are Lie-equivalent if there exists a surjective isomorphism $T: B \rightarrow B^{\prime}$ with $\mathfrak{A}(B)=T^{-1} \mathfrak{A}\left(B^{\prime}\right) T$. If $B$ and $B^{\prime}$ are each $\mathbf{R}^{n}$ with respective norms compatible with $\|\cdot\|_{E_{n}}$, the above proof shows that $B$ and $B^{\prime}$ are Lie-equivalent if and only if $\mathfrak{A}(B)$ and $\mathfrak{A}\left(B^{\prime}\right)$ are orthogonally equivalent. Evidently this equivalence relation is thus an isometric classifying invariant and the problem thus presents itself: classify all n-dimensional Banach spaces up to Lie-equivalence. We discuss the solution for dimensions up to four at the end of section two.

\section{Orthogonal decompositions.}

We pass now to the concept of orthogonal complements in Banach spaces.

Definition. Let $B$ be real or complex with $X$ and $Y$ subspaces of $B . Y$ is said to be an orthogonal complement of $X$ if $X+Y=B$ and if for all $x \in X, y \in Y$ and scalars $\alpha$ and $\beta$ with $|\alpha|=|\beta|=1,\|x+y\|=\| \alpha x+$ $\beta y \|$.

Now suppose $Y$ is an orthogonal complement of $X$. It follows easily from the definition that then both $X$ and $Y$ must be closed. We shall prove momentarily that $Y$ is then unique; hence we may refer to $Y$ as the orthogonal complement of $X$. We shall also refer to the projection $P$ with range $X$ and kernel $Y$ as the orthogonal projection onto $X$. Note that $\|P\|=1$, and $\|I-P\| \leq 1$. We shall denote $Y$ by $\mathcal{O}(X)$. Evidently then $X=\mathcal{O}(\mathcal{O}(X))$. We also note in passing that in the definition of orthogonal complement, it suffices to take $\alpha=1, \beta$ arbitrary. We say that $X$ is orthogonally complemented if $X$ has an orthogonal complement. It is evident that if $B$ is a Hilbert space and $Y$ is an orthogonal complement of $X$, then $Y$ is the Hilbert-space orthogonal complement of $X$.

Proposition 1.5. Suppose $B$ has a compatible Euclidean norm $\|\cdot\|_{H}$ and $X$ is a subspace with an orthogonal complement $Y$. Then $Y$ is the orthogonal complement of $X$ in the Hilbert space $\left(B,\|\cdot\|_{H}\right)=H$.

Proof. For each scalar $\beta$ with $|\beta|=1$, define $U_{\beta}: B \rightarrow B$ by $U_{\beta}(x+y)=x+\beta y$ for all $x \in X$ and $y \in Y$. Evidently $U_{\beta} \in \mathscr{I}(B)$ for all such $\beta$, hence $U_{\beta} \in \mathscr{I}(H)$. The conclusion now follows immediately.

Our next result yields the uniqueness of orthogonal complements.

Proposition 1.6. Let $X_{1}, X_{2}, Y_{1}, Y_{2}$ be subspaces of $B$ so that $Y_{i}$ is an orthogonal complement for $X_{i}$ for $i=1,2$ and $X_{1} \subset X_{2}$. Then $Y_{1} \supset Y_{2}$. 
Proof. It suffices to prove this for real Banach spaces, since if $Y$ is a "complex" orthogonal complement for $X, Y$ is a "real" orthogonal complement. Define then isometries $U_{i}: B \rightarrow B$ by $U_{i}(x+y)=x-y$ for all $x \in X_{i}$ and $y \in Y_{i}$; also define $P_{i}=\left(U_{i}+I\right) / 2$ for $i=1$, 2. It is evident that $P_{i}$ is a projection onto $X_{i}$ with null space $Y_{i}$; also $U_{i}^{2}=I$ for $i=1,2$. It is also noteworthy that our assertion is equivalent to the assertion that $P_{1}$ and $P_{2}$ commute (or that $U_{1}$ and $U_{2}$ commute). Suppose it were false that $Y_{1} \supset Y_{2}$. We could then choose a $b \in Y_{2}$ with $P_{1}(b) \neq 0$. Now let $Z=\left[b, P_{1}(b)\right]$. Then $Z$ is invariant under both $P_{1}$ and $P_{2}$; indeed, $P_{1}(Z)=\left[P_{1}(b)\right]=P_{2}(Z)$. Since $Z$ is (at most) two-dimensional, it has a compatible Euclidean norm, whence it follows that since $Z$ is invariant under both $U_{1}$ and $U_{2}, U_{1} \mid Z$ and $U_{2} \mid Z$ commute. Or put another way, $P_{i} \mid Z$ is simply the orthogonal projection of $Z$ onto $\left[P_{1}(b)\right]$ in the Euclidean norm for $i=1,2$. Hence $P_{1}\left|Z=P_{2}\right| Z$ which is absurd since $P_{1} b \neq 0$ while $P_{2} b=0$.

It is evident that if $X \subset Y$ are subspaces of $B$ with $X$ orthogonally complemented in $B$, then $X$ is orthogonally complemented in $Y$ and then its orthogonal complement relative to $Y$ is simply $\mathcal{O}(X) \cap Y$. Moreover $\mathcal{O}(X) \cap Y$ is orthogonally complemented in $B$, by $X+\mathcal{O}(Y)$. It is not true in general that $X$ orthogonally complemented in $Y$ and $Y$ orthogonally complemented in $B$ implies $X$ orthogonally complemented in $B$; we shall note certain special cases later, where this occurs. A final bit of terminology: say that subspaces $X$ and $Y$ of $B$ are orthogonal if $\mathcal{O}(X)$ and $\mathcal{O}(Y)$ both exist with $X \subset \mathcal{O}(Y)$ (equivalently, $Y \subset \mathcal{O}(X)$ ). More generally, a family of subspaces is called orthogonal if distinct subspaces in the family are orthogonal. We note that if $X_{1}, \ldots, X_{n}$ are orthogonal subspaces of $B$, then $X_{1}+\cdots+X_{n}$ is orthogonally complemented with $\mathcal{O}\left(X_{1}+\cdots+X_{n}\right)=\bigcap_{i=1}^{n} \mathcal{O}\left(X_{i}\right)$.

We pass now to the concepts of unconditional and functional unconditional decompositions.

Definition. Let $\left(X_{\alpha}\right)_{\alpha \in \Lambda}$ be a family of nonzero closed linear subspaces of a real or complex Banach space $X$ such that $\left[X_{\alpha}\right]_{\alpha \in \Lambda}=X$. $\left(X_{\alpha}\right)$ is called a one-unconditional (resp. functional unconditional) decomposition of $X$ if for all $n, \alpha_{1}, \ldots, \alpha_{n}$ distinct members of $\Lambda$ and $x_{i}, x_{i}^{\prime}$ in $X_{\alpha_{i}}$ so that there are scalars $\alpha_{i}$ with $\left|\alpha_{i}\right|=1$ and $x_{i}^{\prime}=\alpha_{i} x_{i}$ (resp. so that $\left.\left\|x_{i}^{\prime}\right\|=\left\|x_{i}\right\|\right)$ for all $i,\left\|\sum_{i=1}^{n} x_{i}\right\|=\left\|\sum_{i=1}^{n} x_{i}^{\prime}\right\|$. If $\left(x_{\alpha}\right)_{\alpha \in \Lambda}$ are given elements of $X,\left(x_{\alpha}\right)_{\alpha \in \Lambda}$ is a one-unconditional basis of $X$ if $\left(\left[x_{\alpha}\right]\right)_{\alpha \in \Lambda}$ is a one-unconditional decomposition of $X$.

Evidently $\left(X_{\alpha}\right)_{\alpha \in \Lambda}$ is a functional unconditional decomposition of $X$ precisely when for any $x=\sum_{\alpha \in \Lambda} x_{\alpha}$, the norm of $x$ is a function of the 
norms of the $x_{\alpha}$ 's. We recall that $\left[X_{\alpha}\right]_{\alpha \in \Lambda}$ is called an unconditional decomposition of $X$ if for all $x$ in $X$, there is a unique family $\left(x_{\alpha}\right)_{\alpha \in \Lambda}$ with $x_{\alpha} \in X_{\alpha}$ for all $\alpha$ and $\sum_{\alpha \in \Gamma} x_{\alpha}$ converging unconditionally to $x$. (This means, $\lim _{\mathscr{D}} \sum_{\alpha \in F} x_{\alpha}=x$, where $\mathfrak{D}$ is the set of all finite subsets of $\Gamma$ directed by inclusion. Equivalently, countably many of the $x_{\alpha}$ 's, say $x_{\alpha_{1}}$, $x_{\alpha_{2}}, \ldots$ are nonzero and for all permutations $\sigma$ of $N, \sum x_{\alpha_{\sigma(i)}}$ converges to $x$.) It is well known that if $\left[X_{\alpha}\right]_{\alpha \in \Lambda}$ is an unconditional decomposition of $X$, then $X$ can be renormed so that $\left(X_{\alpha}\right)_{\alpha \in \Lambda}$ is a one-unconditional decomposition.

We omit the proof of the following simple but basic result.

Proposition 1.7. Let $X,\left(X_{\alpha}\right)_{\alpha \in \Lambda}$ be nonzero Banach spaces.

(a) Suppose $X_{\alpha} \subset X$ for all $\alpha$ and $\left[X_{\alpha}\right]_{\alpha \in \Lambda}=X$. Then $\left(X_{\alpha}\right)$ is a one-unconditional decomposition of $X$ if and only if $X_{\alpha}$ is orthogonal to $X_{\beta}$ for all $\alpha \neq \beta$ (and then for all $\left.\alpha, \mathcal{O}\left(X_{\alpha}\right)=\left[X_{\beta}\right]_{\beta \neq \alpha}\right)$.

(b) $X$ is a functional unconditional sum of $\left(X_{\alpha}\right)_{\alpha \in \Lambda}$ if and only if there exists a functional unconditional decomposition $\left(X_{\alpha}^{\prime}\right)_{\alpha \in \Lambda}$ of $X$ with $X_{\alpha}$ isometric to $X_{\alpha}^{\prime}$ for all $\alpha$.

Functional unconditional sums are defined in the introduction. In view of 1.7(a), we also term one-unconditional decompositions orthogonal decompositions. It is worth pointing out that if $\left(X_{\alpha}\right)_{\alpha \in \Lambda}$ is a functional unconditional decomposition of $X$, we may take arbitrary $u_{\alpha} \in X_{\alpha}$ with $\left\|u_{\alpha}\right\|=1$ for all $\alpha$ and set $\underline{u}=\left(u_{\alpha}\right)_{\alpha \in \Lambda}, U=\left[u_{\alpha}\right]_{\alpha \in \Lambda}$; then $X$ is canonically isometric to $\left(\Sigma_{\Lambda} \oplus X_{\alpha}\right)_{\underline{\underline{u}}}$.

We next present a criterion which insures that a family of orthogonally complemented subspaces have an orthogonally complemented closed linear span. Recall that $c_{0}$ denotes the Banach space of sequences vanishing at infinity.

Proposition 1.8. Let $\left(X_{\alpha}\right)_{\alpha \in D}$ be a family of orthogonally complemented subspaces of $B$, directed by inclusion. (That is, $D$ is a directed set, where for $\alpha, \beta$ in $D, \alpha \leq \beta$ if and only if $X_{\alpha} \subset X_{\beta}$.) Let $X=\left[X_{\alpha}\right]_{\alpha \in D}$ and assume $c_{0}$ does not embed in $X$. Then $X$ is orthogonally complemented with $\mathcal{O}(X)=\bigcap_{\alpha \in D} \mathcal{O}\left(X_{\alpha}\right)$.

We first draw an easy consequence.

COROLlaRY 1.9. Let $\left(Y_{\alpha}\right)_{\alpha \in \Gamma}$ be an orthogonal family of subspaces of $B$ so that $c_{0}$ does not embed in $Y=\left[Y_{\alpha}\right]_{\alpha \in \Gamma}$. Then $Y$ is orthogonally complemented with $\mathcal{O}(Y)=\bigcap_{\alpha \in \Gamma} \mathcal{O}\left(Y_{\alpha}\right)$. 
Proof. Let $D$ be the family of all finite nonempty subsets of $\Gamma$, directed by inclusion. For each $F$ in $D$, let $X_{F}=\Sigma_{\alpha \in F} Y_{\alpha}$; now apply Proposition 1.8.

Proof of 1.8. Let $W=\bigcap_{\alpha \in D} \mathcal{O}\left(X_{\alpha}\right)$ and $Z=X+W$. It is easily seen that $X$ is orthogonally complemented in $Z$ with orthogonal complement $W$. We must show $Z=X$. For each $\alpha$ in $D$, let $P_{\alpha}$ be the orthogonal projection onto $X_{\alpha}$. Note that if $\alpha \leq \beta$, then $P_{\alpha} P_{\beta}=P_{\beta} P_{\alpha}=P_{\alpha}$.

We prove the following claim:

$$
\lim _{\alpha \in D} P_{\alpha} b \text { exists for all } b \text { in } B .
$$

Once this is established, define $P$ by $P b=\lim _{\alpha \in D} P_{\alpha} b$ for all $b \in B$. Then $P$ is a projection with range $X$ and $W$ is clearly contained in its null space. Suppose $P b=0$ yet $b \notin W$. Choose $\alpha_{0}$ with $b \notin \mathcal{O}\left(X_{\alpha_{0}}\right)$. Hence $P_{\alpha_{0}} b \neq 0$. Now choose $\alpha \geq \alpha_{0}$ with $\left\|P_{\alpha} b\right\|<\left\|P_{\alpha_{0}} b\right\|$. But $P_{\alpha_{0}} b=P_{\alpha_{0}} P_{\alpha} b$, and $\left\|P_{\alpha_{0}}\right\|=1$, so $\left\|P_{\alpha_{0}} b\right\| \leq\left\|P_{\alpha} b\right\|$, a contradiction.

To establish (4), we need only prove, fixing $b$ in $B$, that $\left(P_{\alpha} b\right)_{\alpha \in D}$ is a Cauchy net. Were this false, we could choose $\delta>0$ and $\alpha_{1}<\alpha_{2}<\cdots$ in $D$ with $\left\|\left(P_{\alpha_{j+1}}-P_{\alpha_{j}}\right) b\right\|>\delta$ for all $j$. For each $j$, let $Y_{j}=$ $\left(P_{\alpha_{j}}-P_{\alpha_{J-1}}\right) B$ (where $P_{\alpha_{0}}=0$ by definition). It follows that $\left(Y_{j}\right)$ is a one-unconditional decomposition for its closed linear span, since in fact the $Y_{j}$ 's are orthogonal subspaces of $B$. Now setting $y_{j}=\left(P_{\alpha_{j}}-P_{\alpha_{j-1}}\right) b$ for all $j$, we have that $\sum_{j=1}^{n} y_{j}=P_{\alpha_{n}} b$, hence $\left\|\sum_{j=1}^{n} y_{j}\right\| \leq\|b\|$ for all $n$. Since $c_{0}$ does not embed in $X$, the series converges (i.e., $\left(Y_{j}\right)$ is boundedly complete), hence $\left\|y_{j}\right\| \rightarrow 0$, a contradiction.

REMARK. Corollary 1.9 may be strengthened as follows: suppose $\left(Y_{\alpha}\right)_{\alpha \in \Gamma}$ is an orthogonal family of subspaces of $B$ with $Y=\left[Y_{\alpha}\right]_{\alpha \in \Gamma \text {. }}$. Suppose for any family $\left(y_{\alpha}\right)_{\alpha \in \Gamma}$ with $y_{\alpha} \in Y_{\alpha}$ for all $\alpha, c_{0}$ does not

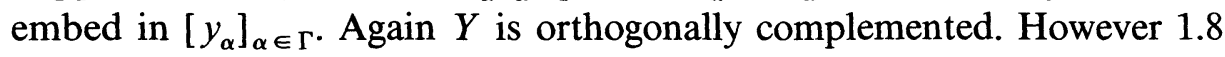
and 1.9 fail in general, if no " $c_{0}$ " assumption is made. Indeed, let $B=l^{\infty}$ (the space of all bounded sequences of scalars) and let $X_{n}=\left\{g \in l^{\infty}\right.$ : $g(j)=0$ for all $j>n\}$. Then $X_{n}$ is orthogonally complemented for all $n$. If $X=\bar{U}_{n}$ and $Y=X+[1]$, then $X=c_{0}$ is not orthogonally complemented in $Y$ and $X$ is not complemented in $B$.

We conclude our discussion of orthogonality in establishing a result connecting this with the Lie algebra of a Banach space. We call this result the "Diagonalization Theorem." (This is a straightforward analogue of the known result for complex Banach spaces [6].) 
THEOREM 1.10. Let $\left(X_{\alpha}\right)_{\alpha \in \Lambda}$ be a one-unconditional decomposition of $B$ with corresponding orthogonal projections $\left(P_{\alpha}\right)_{\alpha \in \Lambda}$, and let $T$ be skewHermitian on $B$. Then $\sum_{\alpha \in \Lambda} P_{\alpha} T P_{\alpha}$ converges unconditionally in the strong operator topology to a skew-Hermitian operator $S$ with $\|S\| \leq\|T\|$.

It suffices to prove this for a real space $B$, for the real case implies the complex one. We first prove the result when $\Lambda$ has two elements. In other words, if $P$ is an orthogonal projection on $B$ and $Q=I-P$, then $P T P+Q T Q$ is skew-Hermitian. Since $P$ is an orthogonal projection, the operator $U=P-Q$ belongs to $\mathscr{I}(B)$ and $(P-Q)^{-1}=P-Q$; hence $(P-Q) T(P-Q)$ is skew-Hermitian (Proposition 1.2(a)). Also $T=(P+Q) T(P+Q)$ is skew-Hermitian. Hence $P T P+Q T Q=$ $\frac{1}{2}[T+(P-Q) T(P-Q)]$ is skew-Hermitian. (This case was done for motivation only.)

Next, suppose $\Lambda$ has $n$ elements, say $\Lambda=\{1, \ldots, n\}$. Let $\varepsilon_{1}, \ldots, \varepsilon_{n}$ be given with $\varepsilon_{i}= \pm 1$ for all $i$. Thus $P_{1}+\cdots+P_{n}=I$ and $\varepsilon_{1} P_{1}$ $+\cdots+\varepsilon_{n} P_{n}$ is a self-inverse isometry of $X$, so again by Proposition 1.2(a),

$$
\left(\varepsilon_{1} P_{1}+\cdots+\varepsilon_{n} P_{n}\right) T\left(\varepsilon_{1} P_{1}+\cdots+\varepsilon_{n} P_{n}\right)
$$

is skew-Hermitian. Expanding (5), we obtain

$$
P_{1} T P_{1}+\cdots+P_{n} T P_{n}+\sum_{i \neq j} \varepsilon_{i} \varepsilon_{j} P_{i} T P_{j}
$$

Since $\mathfrak{A}(B)$ is a linear space, the average $S$ of all operators of the form (6), over all choices of signs $\varepsilon=\left(\varepsilon_{1}, \ldots, \varepsilon_{n}\right)$, is skew-Hermitian. We have that

$$
S=P_{1} T P_{1}+\cdots+P_{n} T P_{n}+\sum_{i \neq j} \frac{1}{2^{n}} \sum_{\varepsilon} \varepsilon_{i} \varepsilon_{j} P_{i} T P_{j}
$$

where the second sum extends over all choices of signs. But for $i \neq j$,

$$
\frac{1}{2^{n}} \sum_{\varepsilon} \varepsilon_{i} \varepsilon_{j}=\int_{0}^{1} r_{i}(\omega) r_{j}(\omega) d \omega=0
$$

where $r_{1}, \ldots, r_{n}$ denote the first $n$ Rademacher functions. Hence $S=$ $P_{1} T P_{1}+\cdots+P_{n} T P_{n}$ is skew-Hermitian. This proof also showed that $\|S\| \leq\|T\|$. It thus follows incidentally that

$$
\left\|P_{1} T P_{1}+\cdots+P_{n-1} T P_{n-1}\right\| \leq\|T\| .
$$

Indeed, if $Q=I-\left(P_{1}+\cdots+P_{n-1}\right)$, then $P_{1} T P_{1}+\cdots+P_{n-1} T P_{n-1}=$ $Q S Q$. 
Now to obtain Theorem 1.10 , let $D$ be the family of all finite nonempty subsets of $\Lambda$, directed by inclusion. For each $F \in D$, define $Q_{F}=I-\sum_{\alpha \in F} P_{\alpha}, S_{F}=\sum_{\alpha \in F} P_{\alpha} T P_{\alpha}+Q_{F} T Q_{F}$, and $T_{F}=\sum_{\alpha \in F} P_{\alpha} T P_{\alpha}$. It follows that $Q_{F} B \cup\left\{X_{\alpha}: \alpha \in F\right\}$ is a one-unconditional decomposition of $B$ and hence we have that $S_{F}$ is skew-Hermitian with

$$
\left\|S_{F}\right\| \leq\|T\|, \quad\left\|T_{F}\right\| \leq\|T\| .
$$

Now let $X$ denote the linear span of the $X_{\alpha}$ 's. We have easily that for any $b \in X$.

$$
\lim _{F \in D} S_{F}(b)=\lim _{F \in D} T_{F}(b)
$$

exists. Since $X$ is dense in $B,(8)$ and (9) yield the existence of a unique $S \in \mathscr{L}(B)$ with $\|S\| \leq\|T\|$ and

$$
S b=\lim _{F \in D} S_{F}(b)=\lim _{F \in D} T_{F}(b) \quad \text { for all } b \in B .
$$

Since $\mathfrak{A}(B)$ is closed in the strong operator topology, $S$ is skew-Hermitian, completing the proof.

REMARK. The argument for 1.10 also shows that if $T$ is an arbitrary operator on $B$, so is $S=\sum_{a \in \Lambda} P_{a} T P_{a}$ and $\|S\| \leq\|T\|$. This result, for the case of one-unconditional bases, is due to A. E. Tong [13].

\section{Well-embedded Hilbert spaces and Hilbert components.}

Let $X$ be a subspace of $B . X$ is said to be well-embedded in $B$ if there is a subspace $Y$ of $B$ so that $B=X+Y$ and for all $x \in X, y \in Y$, and $U \in \mathscr{I}(B),\|x+y\|=\|U x+y\|$. It follows immediately that $X$ is orthogonally complemented with $Y=\mathcal{O}(X)$; thus $Y$ is uniquely determined. It also follows that if $Y$ is well-embedded in $B$ and $X$ is a subspace of $Y$ with $X$ orthogonally complemented in $Y$, then $X$ is orthogonally complemented in $B . X$ is said to be a well-embedded Hilbert space if $X$ is Euclidean and well-embedded. Evidently any one-dimensional orthogonally complemented subspace is thus a well-embedded Hilbert space. Finally, $X$ is said to be a Hilbert component of $B$ if $X$ is a maximal nonzero well-embedded Hilbert subspace.

REMARK. The concept of a well-embedded Hilbert subspace is introduced for complex spaces in [14]; that of a Hilbert component in [6]. Our definition is quite different from that in [6]; we will show the desired equivalence later.

Our next result gives some simple equivalences to this concept. 
Proposition 1.11. Let $X$ be a nonzero Euclidean subspace of $B$ and $Z$ a subspace such that $X \oplus Z=B$. Also assume $\operatorname{dim} X>1$ if $B$ is real. The following are equivalent:

(1) $X$ is a well-embedded Hilbert subspace with $\mathcal{O}(X)=Z$.

(2) For all $x, x^{\prime}$ in $X$ and $z$ in $Z$, if $\|x\|=\left\|x^{\prime}\right\|$ then $\|x+z\|=$ $\left\|x^{\prime}+z\right\|$.

(3) For all $T \in \mathfrak{U}(X), T \oplus 0 \in \mathfrak{A}(B)$.

Remark. $B=X \oplus Z$ means $B=X+Z$ and $X \cap Z=\{0\}$. When $B=X \oplus Z$ and $T \in \mathscr{L}(X), S \in \mathscr{L}(Z), T \oplus S$ is defined by $T \oplus$ $S(x \oplus z)=T x+S z$ for all $x$ in $X$ and $z$ in $Z$.

Proof. (1) $\Rightarrow(3)$ : Let $T \in \mathfrak{A}(X)$ and $x$ be a real number. Then $e^{x T} \in \mathscr{I}(X)$, hence $e^{x T} \oplus I \in \mathscr{I}(B)$. But of course $e^{x T} \oplus I=e^{x \tilde{T}}$ where $\tilde{T}=T \oplus 0$, hence we obtain $\tilde{T} \in \mathfrak{A}(B)$.

(3) $\Rightarrow(2)$. Let $x$ and $x^{\prime}$ in $X$ with $\|x\|=\left\|x^{\prime}\right\|$ and $z \in Z$. We may assume of course that $\|x\|=1$. Now if $\operatorname{dim} X \geq 2$, choose a two-dimensional subspace $Y$ of $X$ with $x, x^{\prime} \in Y$. Then it follows that there is a rank-two $T \in \mathfrak{A}(X)$ with $T X \subset Y$ such that $e^{T} x=x^{\prime}$. Since $\tilde{T}=T \oplus 0$ $\in \mathfrak{A}(B), e^{\tilde{T}} \in \mathscr{I}(B)$, hence $\left\|e^{\tilde{T}}(x+z)\right\|=\left\|x^{\prime}+z\right\|$ and $e^{\tilde{T}}(x+z)=x^{\prime}$ $+z$. If $B$ is complex and $X$ is one-dimensional, let $T y=i y$ for $y \in X$. Then $T \in \mathfrak{A}(Y)$. Choose $\theta, 0 \leq \theta<2 \pi$ so that $x^{\prime}=(\cos \theta+i \sin \theta) x$; then $e^{\theta T} x=x^{\prime}$ and so as before, $\|x+z\|=\left\|x^{\prime}+z\right\|$.

(2) $\Rightarrow(1)$. Let $U \in \mathscr{I}(X)$ and fix $x \in X$ and $z \in Z$. Then setting $x^{\prime}=U x$, we have that $(U \oplus I)(x+z)=x^{\prime}+z$ and $\|x\|=\left\|x^{\prime}\right\|$, whence $\|(U \oplus I)(x+z)\|=\left\|x^{\prime}+z\right\|$, so $U \oplus I \in \mathscr{I}(B)$.

We may now easily prove the existence of Hilbert components.

TheOREM 1.12. Let $X$ be a well-embedded Hilbert subspace of $B$. Then there exists a Hilbert component of $B$ containing $X$.

Proof. If there does not exist a well-embedded Hilbert space $Y \supset X$ with $\operatorname{dim} Y \geq 2$, the result is trivial. Otherwise, let (S) denote the family of all well-embedded Hilbert subspaces $Y$ of $B$ with $Y \supset X$ and $\operatorname{dim} Y \geq 2$. We order (S) by inclusion and apply Zorn's Lemma to show that $\mathbb{S}$ has a maximal element. Let $\mathfrak{N}$ be a nest in $\mathfrak{S}$. Thus, we may set $\mathfrak{N}=\left\{X_{\alpha}\right.$ : $\alpha \in \Lambda\}$ where for all $\alpha$ and $\beta \in \Lambda, Y_{\alpha} \subset Y_{\beta}$ or $Y_{\beta} \subset Y_{\alpha}$ and $Y_{\alpha}$ is a well-embedded Hilbert subspace of $B$ containing $X$. Now let $Y=\cup_{\alpha \in \Lambda} Y_{\alpha}$. Since the parallelogram identity holds on $Y$ (or the polarization identity if $B$ is complex), we have that $\bar{Y}$ is Euclidean. In particular, $c_{0}$ does not 
embed in $\bar{Y}$. Then setting $Z=\bigcap_{\alpha \in \Lambda} \mathcal{O}(Y)$, we have that $\bar{Y}$ is orthogonally complemented by $Z$, by Proposition 1.8. Finally, we have that if $x, x^{\prime}$ are in $Y$ and $z$ is in $Z$, there is an $\alpha$ so that $x, x^{\prime}$ are in $Y_{\alpha}$. Assuming $\|x\|=\left\|x^{\prime}\right\|$, since $z \in \mathcal{O}\left(Y_{\alpha}\right)$, we have that $\|x+z\|=\left\|x^{\prime}+z\right\|$. It now follows easily that the same conclusion holds for $x, x^{\prime}$ in $\bar{Y}$ with $\|x\|=\left\|x^{\prime}\right\|$, proving that $\bar{Y}$ is indeed a well-embedded Hilbert subspace of $B$, by Proposition 1.11 .

REMARK. Our proof is valid over both real and complex spaces. However the complex-case is easily deduced from the real one alone. Indeed, suppose $B$ is a complex Banach space and $H$ is a Hilbert component of $B_{\mathbf{R}}$. We claim that then $H$ is a Hilbert component of $B$. Evidently it suffices to prove that $H$ is well-embedded in $B$. In fact it is enough to show that $\mathcal{O}(H)$ is a complex orthogonal complement for $H$. Define $T$ on $B_{\mathbf{R}}$ by $T b=i b$ for all $b \in B$. Then $T$ is skew-Hermitian on $B_{\mathrm{R}}$; hence (as we show in Proposition 3.2), $T H \subset H$. Thus $H$ is a subspace of $B$. Since $T \in \mathscr{I}\left(B_{\mathbf{R}}\right)$, we have that $T \mathcal{O}(H)=\mathscr{O}(T H)=$ $\mathcal{O}(H)$ by uniqueness of orthogonal complements. Thus both $H$ and $\mathcal{O}(H)$ are complex linear and of course $H$ is a complex Hilbert space. Finally, if $\alpha$ and $\beta$ are real numbers with $\alpha^{2}+\beta^{2}=1$, then $(\alpha I+\beta T) \mid H \in \mathscr{I}(H)$ and hence for all $h \in H$ and $y \in \mathcal{O}(H)$, $\|(\alpha I+\beta T) h+y\|=\|h+y\|$ since $H$ is well-embedded; but this shows $H$ is orthogonally complemented by $\mathcal{O}(H)$ in $B$, completing the argument.

\section{Characterizations of well-embedded Hilbert subspaces and the struc-} ture of Hilbert components. To first summarize the main results of this section, we introduce the following natural isometric invariant:

Definition. Let $X$ be a subspace of $B$ and set

$$
\begin{aligned}
& \mathfrak{U}^{\prime}(X, B)=\{T \in \mathfrak{A}(B): T B \subset X\}, \\
& \mathfrak{U}^{\prime}(X)=\left\{T \mid X: T \in \mathfrak{U}^{\prime}(X, B)\right\} .
\end{aligned}
$$

Evidently $\mathfrak{A}^{\prime}(X, B)$ is a Lie subalgebra of $\mathfrak{A}(B)$ and by the HahnBanach theorem, $\mathfrak{A}^{\prime}(X)$ is a Lie subalgebra of $\mathfrak{A}(X)$. Moreover the natural map $T \rightarrow T \mid X$ from $\mathfrak{U}^{\prime}(X, B)$ to $\mathfrak{U}^{\prime}(X)$ is clearly real-linear, norm-contractive and surjective. We claim that this map is one-one (hence $\mathfrak{A}^{\prime}(X)$ and $\mathfrak{A}^{\prime}(X, B)$ are isomorphic vector spaces). Indeed, suppose $T \in \mathfrak{U}^{\prime}(X, B)$ and $T \mid X=0$. Then $T^{2}=0$. Hence for any real $x, e^{x T}=$ $I+x T$. But $\left\|e^{x T}\right\|=1$ since $T$ is skew-Hermitian. Were $T \neq 0$, $\lim _{x \rightarrow \infty}\|I+x T\|=\infty$, a contradiction. 
The next three theorems constitute the main results of section two.

TheOREM 2.1. Let $B$ be real, $n \geq 2$ and $X$ an $n$-dimensional subspace of $B$.

(a) If $\operatorname{dim} \mathfrak{X}^{\prime}(X)>(n-1)(n-2) / 2$, then $X$ is a well-embedded Hilbert subspace of $B$ (and then $\left.\operatorname{dim} \mathfrak{A}^{\prime}(X)=n(n-1) / 2\right)$.

(b) $\operatorname{dim} \mathfrak{Y}^{\prime}(X)=(n-1)(n-2) / 2$ if and only if $X$ contains an $n-1$ dimensional well-embedded Hilbert subspace but $X$ is not itself a well-embedded Hilbert space.

Theorem 3 of the introduction follows easily from this result, upon setting $B=X$. Indeed, (a) is immediate. To see (b), suppose $\operatorname{dim} \mathfrak{A}(X)=$ $(n-1)(n-2) / 2$. Then 2.1 (b) implies $X$ is non-Euclidean, yet has a codimension-one well-embedded Hilbert subspace $Y$. Hence the orthogonal complement $Z$ of $Y$ is one-dimensional; it follows from Proposition 1.11 that $X$ is a functional unconditional sum of $Y$ and $Z$, hence $X$ is a non-Euclidean rotation space. It follows immediately from the Diagonalization Theorem 1.10 that if $X$ is a non-Euclidean rotation space, $\operatorname{dim} \mathfrak{A}(X) \geq(n-1)(n-2) / 2$; hence by part (a), $\operatorname{dim} \mathfrak{U}(X)=$ $(n-1)(n-2) / 2$. (However we shall find it more convenient to establish Theorem 3 of the Introduction first.)

Evidently if $X$ is a well-embedded Hilbert subspace of $B$, then every subspace $Y$ of $X$ is also a well-embedded Hilbert subspace of $B$. In particular, every one-dimensional subspace of $X$ is orthogonally complemented in $B$. The next result shows the converse is true.

THEOREM 2.2. Suppose $X$ is a subspace of $B$ and every one-dimensional subspace of $X$ is orthogonally complemented in $B$. Then $X$ is a well-embedded Hilbert subspace of $B$.

REMARKS. 1. If $X=B$ itself, this gives a (known) isometric characterization of Hilbert space. (For real $B$, this is due to Saint-Raymond; for complex $B$, to Berkson.)

2. The result is known for complex $B$ (although not stated in this way) and due to Kalton and Wood [6]. Indeed, assuming $B$ is complex, suppose $P$ is a projection on $B$. It follows from the equivalences in Theorem 1.1 that $P$ is an orthogonal projection if and only if $P$ is Hermitian (this result is due to Berkson [1]). It follows that if $b$ is a nonzero element of $B,[\mathrm{~b}]$ is orthogonally complemented if and only if $b$ is in the range of a rank-one Hermitian operator on $B$; such elements (together with 0 ) are termed Hermitian elements of $B$. Kalton and Wood 
term $X$ a Hilbert component provided $X$ is a maximal linear subspace of the Hermitian elements. It is then proved in [6] that Hilbert components are well-embedded Hilbert subspaces; hence our definition is equivalent to that in [6] for complex Banach spaces, taking Theorem 1.12 into account. Notice that we may also phrase 2.2 for complex $B$ as: $X$ is a well-embedded Hilbert subspace provided every nonzero element is in the range of a rank-one skew-Hermitian operator on $B$.

3. Suppose $B$ is real and $X$ is a well-embedded Hilbert subspace of $B$ of dimension at least 2. We then have that $\mathcal{O}(X)=W$, where $W=$ $\bigcap\{\operatorname{ker} T: T B \subset X, \operatorname{rank} T=2$ and $T \in \mathfrak{A}(B)\}$. (Throughout, $\operatorname{ker} T=$ $\{b: T b=0\}=$ null space $T$.) Indeed, suppose first $T \in \mathfrak{A}(B)$, rank $T=2$, $T B \subset X$. Since $T B$ is well-embedded, it is orthogonally complemented, and $\mathcal{O}(T B) \supset \mathcal{O}(X)$ since $T B \subset X$. But $\mathcal{O}(T B)=\operatorname{ker} T$; this proves that $\mathcal{O}(X) \subset W$. Next let $w \in W$, and choose $x \in X, z \in \mathcal{O}(X)$ with $w=x \oplus$ $z$. If $x \neq 0$, choose a rank-two member $T$ of $\mathfrak{A}(B)$ with $x \in T B \subset X$ (possible since $X$ is well-embedded). It follows of course that $T x \neq 0$. Since $\mathcal{O}(X) \subset \mathcal{O}(T B)=\operatorname{ker} T$ as already shown, $T z=0$, whence $T w \neq 0$, contradicting the definition of $W$. Moreover suppose $Y$ is a subspace of $B$ with $\operatorname{dim} Y \geq 2$. Then $Y$ is a well-embedded Hilbert space if (and only if) every element of $Y$ is in the range of a rank-two member of the Lie algebra of $B$. Indeed, suppose $x \in T B$ with $T \in \mathfrak{A}(B)$ and $T$ rank-two. Theorem 2.1(a) applied to $T B$ itself shows that $T B$ is a well-embedded Hilbert space, hence $[x]$ is orthogonally complemented, so this result follows from Theorem 2.2.

THEOREM 2.3. Let $H_{1}$ and $H_{2}$ be distinct Hilbert components of a real $B$ with $H_{1}$ of dimension at least two. Then $H_{1}$ and $H_{2}$ are orthogonal.

It follows immediately from our remark at the end of section one that Hilbert components of a complex Banach space are orthogonal. This result is proved in [6].

The classification under Lie-equivalence of real Banach spaces of dimension up to four is easily completed using Theorem 3 of the Introduction and standard results in the theory of Lie groups; this is given at the end of this section.

Lie algebra characterizations of rotation spaces and well-embedded. Euclidean spaces.

We now begin the detailed work of establishing the three theorems stated above. We first give the proof of the simplest possible case of Theorem 2.1(a). This allows us to set up notation and intuition for the 
following arguments (although we won't use this simplest case explicitly in the sequel). Thus, suppose $B$ is real and $T \in \mathfrak{A}(B)$ is rank-two; set $X=T B$ and $Z=\operatorname{ker} T$. We claim that $X$ is a well-embedded Hilbert space with orthogonal complement $Z$.

Let us introduce the following notation: Define $2 \times 2$ matrices $R_{0}$ and $R(\theta)$ for real $\theta$ by

$$
R_{0}=\left[\begin{array}{cc}
0 & -1 \\
1 & 0
\end{array}\right] \text { and } R(\theta)=\left[\begin{array}{cc}
\cos \theta & -\sin \theta \\
\sin \theta & \cos \theta
\end{array}\right] .
$$

Of course $R_{0} \in \mathrm{SO}_{2} ; R(\theta) \in S \mathrm{O}_{2}$ and $R(\theta)$ is simply the operation of rotating counter-clockwise by $\theta$-radians. A computation yields that

$$
e^{\theta R_{0}}=R(\theta)
$$

(cf. Lemma 2.2 of [10] for a more general result). Now let $T$ be as above and choose a compatible Euclidean norm $\|\cdot\|_{E}$ on $X$. Since then $T \mid X \in$ $\mathfrak{A}\left(X,\|\cdot\|_{E}\right)$, we have that the matrix for $T$ with respect to an orthonormal basis for $X$ equals $c R_{0}$ for some $c \neq 0$. Assume without loss of generality that $c=1$. Since $e^{\theta T} \in \mathscr{I}(B)$ for all $\theta$, we obtain by (11) that $\|\cdot\|_{E}$ and $\|\cdot\|$ are proportional on $X$, so $X$ is Euclidean. Since $T \mid X$ is invertible, we have that $B=X \oplus Z$. It then follows by Proposition 1.11 that $X$ is well-embedded.

The next somewhat technical result allows us to generalize the above reasoning to arbitrary finite-dimensional spaces. It establishes the relationship between $\mathfrak{U}^{\prime}(X)$ and $\mathfrak{U}^{\prime}(X, B)$ for finite-dimensional $X \subset B$. (If $X$ and $Z$ are subspaces of $B$, we write $Y=X \oplus Z$ if $Y$ is a subspace; (i.e., a closed linear submanifold,) and $X \cap Z=\{0\}, X+Z=Y$.)

LEMMA 2.4. Let $X$ be a finite-dimensional subspace of $B$. There exists a subspace $Z$ with $X \oplus Z=B$ so that for all $S \in \mathfrak{U}(X), S \in \mathfrak{U}^{\prime}(X)$ if and only if $S \oplus 0 \in \mathfrak{U}^{\prime}(X, B)$.

Before giving the proof, let us see how this yields the natural characterization of well-embedded Euclidean spaces of finite dimension.

COROLlaRY 2.5. Let $X$ be an $n$-dimensional subspace of $B$ with $n \geq 2$ and assume $B$ is real. $X$ is a well-embedded Hilbert subspace if and only if $\operatorname{dim} \mathfrak{Y}^{\prime}(X)=n(n-1) / 2$.

Proof. One direction is trivial in virtue of the fact that $\operatorname{dim} s 0_{n}=$ $n(n-1) / 2$. Now suppose $\operatorname{dim} \mathfrak{A}^{\prime}(X)=n(n-1) / 2$ and choose $Z$ as in Lemma 2.4; also let $\|\cdot\|_{E}$ be a compatible Euclidean norm on $X$. Fix $x_{0}$ in $X$ with $\left\|x_{0}\right\|=1$ and assume without loss of generality that also 
$\left\|x_{0}\right\|_{E}=1$. Now $\mathfrak{A}^{\prime}(X) \subset \mathfrak{U}(E)$ by Proposition 1.3, where $E=$ $\left(X,\|\cdot\|_{E}\right)$. Since $\operatorname{dim} \mathfrak{A}(E)=n(n-1) / 2, \mathfrak{U}^{\prime}(X)=\mathfrak{A}(E)$. Suppose $x \in$ $E,\|x\|_{E}=1$. By the argument preceding Lemma 2.4 , we may choose a rank-2 $T$ in $\mathfrak{A}(E)$ with $e^{T} x_{0}=x$. But $e^{T} \in \mathscr{I}(B)$, hence $\|x\|=1$, proving that $X$ is Euclidean. Now Proposition 1.11 yields that $X$ is well-embedded.

We pass now to the proof of Lemma 2.4. Of course it suffices to prove the "only if" assertion, the other one being trivial. For each $T$ in $\mathfrak{U}^{\prime}(X, B), \operatorname{ker} T$ is of finite co-dimension, hence $Z^{\prime \prime}=\bigcap\{\operatorname{ker} T: T \in$ $\left.\mathfrak{A}^{\prime}(X, B)\right\}$ is a subspace of finite co-dimension in $B$. (Recall that $\mathfrak{A}^{\prime}(X, B)$ is isomorphic to $\mathfrak{U}^{\prime}(X)$ a finite-dimensional space.) It follows that we may choose a finite-dimensional space $X^{\prime} \supset X$ and a subspace $Z^{\prime}$ of $Z^{\prime \prime}$ with $X^{\prime} \oplus Z^{\prime}=B$. (Let $Z^{\prime}$ be a complementary subspace to $X \cap Z^{\prime \prime}$ in $Z^{\prime \prime}$; $X \oplus Z^{\prime}$ is then of finite co-dimension, so choose $X^{\prime}$ appropriately.) We thus have that

$$
\operatorname{ker} T \supset Z^{\prime} \text { for all } T \in \mathfrak{U}^{\prime}(X, B) \text {. }
$$

Now choose $\|\cdot\|_{E}$ a compatible norm on $X^{\prime}$, let $Y$ be the orthogonal complement to $X$ in $X^{\prime}$ with respect to $\|\cdot\|_{E}$ and finally set $Z=Y \oplus Z^{\prime}$. Now suppose $T \in \mathfrak{A}^{\prime}(X, B)$. Then $T \mid X^{\prime} \in \mathfrak{A}\left(X, X^{\prime}\right)$. Since $T \mid X^{\prime} \in$ $\mathfrak{A}\left(X^{\prime},\|\cdot\|_{E}\right)$, it follows by properties of skew-Hermitian operators on Hilbert space that $T\left|X^{\prime}=T\right| X \oplus 0$ (with respect to the decomposition $X^{\prime}=X \oplus Y$ ). Thus by (12), $T=T \mid X \oplus 0$ (with respect to the decomposition $B=X \oplus Z$ ).

For the concepts involved in the next result, see Theorem 1.4 and the preceding discussion.

Proposition 2.6. Let $X$ be a finite-dimensional subspace of $B$. Then $\mathfrak{U}^{\prime}(X)$ is a $B$. Lie algebra.

Proof. Choose $Z$ as in Lemma 2.4 and let $G^{\prime}=\{U \in \mathscr{I}(X): U \oplus I$ $\in \mathscr{I}(B)\}$ (direct sums refer to the decomposition $B=X \oplus Z$ ). Then $G^{\prime}$ is a closed subgroup of $\mathscr{I}(X)$, hence is a compact Lie group; it follows moreover from Lemma 2.4 that $\mathfrak{U}^{\prime}(X)$ equals the tangent space to $G^{\prime}$ at $I$, hence $\mathfrak{A}^{\prime}(X)$ is the Lie algebra of $G^{\prime}$. By Theorem 1.4, we now need only show that $\mathfrak{U}^{\prime}=\mathfrak{U}^{\prime}(X)$ is saturated. (If $B$ is complex, it's trivial that $i I \mid X \in \mathfrak{U}^{\prime}$.) Let $T \in \mathscr{L}(X)$, and suppose $T x \in \mathfrak{U}^{\prime} x$ for all $x \in X$. Now let $b \in B$ with $b=x+z, x \in X, z \in Z$. Then $(T \oplus 0) b=A x$ for some $A \in \mathfrak{U}^{\prime}$; that is, $(T \oplus 0)(b)=(A \oplus 0)(b)$. By Lemma 2.4, $A \oplus 0 \in \mathfrak{U}(B)$. Since $\mathfrak{A}(B)$ is saturated, $T \oplus 0 \in \mathfrak{U}(B)$, whence $T \in \mathfrak{U}^{\prime}$. 
We pass now to the proof of Theorem 2.1. In fact, it suffices to prove this for the case where $X=B$ itself. Suppose we have done this. Again, let $X$ and $B$ as in the statement of 2.1. For part (a), suppose $\operatorname{dim} \mathfrak{A}^{\prime}(X)>$ $(n-1)(n-2) / 2$. Since $\mathfrak{U}^{\prime}(X)$ is a $B$. Lie algebra, we have that $\operatorname{dim} \mathfrak{A}^{\prime}(X)=n(n-1) / 2$ and hence $X$ is a well-embedded Hilbert space by Corollary 2.5. For part (b), suppose $\operatorname{dim} \mathfrak{U}^{\prime}(X)=(n-1)(n-2) / 2$. Now choose $Z$ as in Lemma 2.4 and choose $Y$ a well-embedded Hilbert subspace of $X$ of dimension $n-1$ (again using the fact that $\mathfrak{A}^{\prime}(X)$ is a $B$. Lie algebra). But then if $W$ is the orthogonal complement of $Y$ in $X$, we have that for any $T \in \mathfrak{A}(Y), \tilde{T} \in \mathfrak{A}(B)$, where $\tilde{T} b=T y$ if $b=y+w$ $+z$ with $y \in Y, w \in W$ and $z \in Z$. Indeed, the set of such $\tilde{T}$ 's equals $\mathfrak{A}^{\prime}(X)$. We have thus shown $\mathfrak{A}(Y)=\mathfrak{A}^{\prime}(Y)$, so $Y$ is well-embedded by Corollary 2.5. (The converse assertion in 2.1(b) is trivial by part (a) and 2.5.)

We are thus reduced to proving Theorem 3 of the Introduction. Part (a) was established in Theorem 3.2 of [10]. We prefer to give a new proof, making use of basic results in Lie groups. Both parts are proved by induction; part (a) is already established for $n=2$ while part (b) follows easily for $n=3$ from our observations preceding Lemma 2.4 (this case is also established in [6]). We first consider part (a); assume $n \geq 3$ and the assertion proved for spaces of smaller dimension. We may of course assume that $B=\left(\mathbf{R}^{n},\|\cdot\|\right)$ with $\|\cdot\|$ and $\|\cdot\|_{E_{n}}$ compatible (recall that $\|\cdot\|_{E_{n}}$ denotes the usual Euclidean norm and $\left.E_{n}=\left(\mathbf{R}^{n},\|\cdot\|_{E_{n}}\right)\right)$. Let $G$ be the component of the identity of $\mathscr{I}(B)$.

We thus have that $G$ is a compact subgroup of $S \mathcal{O}_{n}$ and our assumptions yield that

$$
\operatorname{dim} G=\operatorname{dim} \mathfrak{U}(B)>\frac{(n-1)(n-2)}{2} .
$$

Now fix $x_{0}$ in $S_{n-1}=\left\{x \in \mathbf{R}^{n}:\|x\|_{E_{n}}=1\right\}$ and let $H=\left\{g \in G: g\left(x_{0}\right)\right.$ $\left.=x_{0}\right\}$. Let $Y=x_{0}^{\perp}$ (i.e., $Y$ is the orthogonal complement of $\left[x_{0}\right]$ with respect to the Euclidean norm). Since $H$ is a set of orthogonal matrices, $Y$ is invariant under $H$. Hence if $h \in H$ and $y \in Y, h\left(x_{0}+y\right)=x_{0}+h(y)$. It follows that the Lie algebra to $H$ at $I$ equals $\mathfrak{U}^{\prime}(Y, B)=\mathfrak{U}^{\prime}(Y) \oplus 0$ with respect to the decomposition $B=Y \oplus\left[x_{0}\right]$. Now set $W=G x_{0}$. It follows that $W$ is a compact connected submanifold of $S_{n-1}$. We then claim:

$$
\operatorname{dim} W=n-1 \text {. }
$$

(Here as in (13), "dim" refers to topological dimension.) Once (14) is established, we complete the argument as follows: $W$ must have nonempty interior (relative to $S_{n-1}$ ) by a standard result in topology. But if 
$U \subset W$ is a nonempty open set, so is $G \cdot U=W$, whence $W$ is an open and closed subset of $S_{n-1}$. Since $S_{n-1}$ is connected, $W=S_{n-1}$. It follows that $\|\cdot\|$ is a multiple of $\|\cdot\|_{E_{n}}$ and hence $B$ is Euclidean.

Thus to complete the proof, we may assume that $\operatorname{dim} G x_{0} \neq n-1$ for all $x_{0}$ in $S_{n-1}$ and argue to a contradiction. Now since $W \subset S_{n-1}$, $\operatorname{dim} W \leq n-2$. But by the theory of Lie groups (cf. [5] or [15]),

$$
\operatorname{dim} W=\operatorname{dim} G-\operatorname{dim} H .
$$

Hence we deduce that

$$
\operatorname{dim} H>\frac{(n-1)(n-2)}{2}-(n-2)=\frac{(n-2)(n-3)}{2} .
$$

Since $\operatorname{dim} H=\operatorname{dim} \mathfrak{U}^{\prime}(Y)$ and $\mathfrak{U}^{\prime}(Y)$ is a B. Lie algebra, we have by induction hypothesis that $Y$ is a well-embedded Hilbert subspace of $B$. This proves that any codimension-one subspace of $B$ is a well-embedded Euclidean subspace. Then if $\left(e_{1}, \ldots, e_{n}\right)$ is the usual basis, $Y=\left[e_{2}, \ldots, e_{n}\right]$ is well-embedded and so is $X=\left[e_{1}, e_{2}\right]$. We may assume without loss of generality that $\left\|e_{1}\right\|=1$. Let $Z=\left[e_{3}, \ldots, e_{n}\right], z \in Z$ and $x=a e_{1}+b e_{2}$ for some scalars $a$ and $b$. Then

$$
\begin{aligned}
\|x+z\| & =\|\| x\left\|e_{1}+z\right\| \quad \text { since } X \text { is well-embedded } \\
& =\|\| x\left\|e_{1}+\right\| z\left\|e_{2}\right\| \quad \text { since } Y \text { is well-embedded } \\
& =\left(\|x\|^{2}+\|z\|^{2}\right)^{1 / 2} \quad \text { since } X \text { is Euclidean. }
\end{aligned}
$$

Evidently we have proved $B$ is Euclidean (so of course (14) holds after all).

We prove (b) of Theorem 3 by the same method. The case $n=3$ follows easily from our discussion of the simplest case of 2.1(a). Now assume $n>3$ and the result proved for spaces of smaller dimension; assume then $B$ is $n$-dimensional and $\operatorname{dim} \mathfrak{A}(B)=(n-1)(n-2) / 2$. (The same assumptions regarding $E_{n}$ and $B$ are in force.) Let $G$ be as above and for each $x_{0}$ in $S_{n-1}$, let $H x_{0}=\left\{g \in G: g\left(x_{0}\right)=x_{0}\right\}$. If $Y x_{0}=x_{0}^{\perp}$, the argument in part (a) showed that

$$
\operatorname{dim} \mathfrak{U}^{\prime}\left(Y x_{0}\right)=\operatorname{dim} H x_{0}=\operatorname{dim} G-\operatorname{dim} W x_{0}
$$

where $W x_{0}=G x_{0}$; also $\operatorname{dim} G=(n-1)(n-2) / 2$ by assumption.

We now claim that

$$
\operatorname{dim} H x_{0} \geq \frac{(n-2)(n-3)}{2} .
$$


Indeed, were this false, we obtain by (16) that

$$
\operatorname{dim} W x_{0}>\frac{(n-1)(n-2)}{2}-\frac{(n-2)(n-3)}{2}=n-2 .
$$

Hence $\operatorname{dim} W x_{0}=n-1$, so $W x_{0}=S_{n-1}$ by our argument above. Thus we obtain $\|\cdot\|$ is a multiple of $\|\cdot\|_{E}$ as before, so $B$ is Euclidean. But then $\operatorname{dim} \mathfrak{A}(B)=n(n-1) / 2$, a contradiction.

Now suppose $\operatorname{dim} H x_{0}>(n-2)(n-3) / 2$. Since $Y x_{0}$ is an $n-1$ dimensional space, we thus obtain by (16) that $Y x_{0}$ is a well-embedded Euclidean subspace by part (a) of Theorem 2.3 (which is now established) and hence in particular $\operatorname{dim} \mathfrak{U}^{\prime}\left(Y x_{0}\right)=(n-1)(n-2) / 2$. Then of course $\mathfrak{A}^{\prime}\left(Y x_{0}\right)=\mathfrak{A}(B)$ and $(b)$ is established.

We are now reduced to proving that there exists an $x_{0}$ with $\operatorname{dim} H x_{0}$ $>(n-2)(n-3) / 2$. If this were false, then by (17), we would have that $\operatorname{dim} H x_{0}=(n-2)(n-3) / 2$ for all $x_{0}$ in $S_{n-1}$. This is equivalent to

$$
\operatorname{dim} \mathfrak{A}^{\prime}(Y)=\frac{(n-2)(n-3)}{2}
$$

for all $n$ - 1-dimensional subspaces $Y$ of $B$.

But now it follows by induction hypothesis that any such $Y$ contains a Hilbert subspace of dimension $n-2$, well-embedded in $Y$, since $\mathfrak{A}^{\prime}(Y)$ is a $B$. Lie algebra. Fix $Y$ and suppose $Z$ is such a subspace. Then if $X_{1}=Y^{\perp}$ and $X_{2}=Y \cap Z^{\perp}, X_{1}$ and $X_{2}$ are one-dimensional spaces with $X_{1} \oplus X_{2} \oplus Z=B$ and for any $T, T \in \mathfrak{A}^{\prime}(Y)$ if and only if there is an $S \in \mathfrak{A}(Z)$ so that $T\left(x_{2}+z\right)=S(z)$ for all $x_{2} \in X_{2}$ and $z \in Z$. But $T X_{1}=\{0\}$ if $T \in \mathfrak{U}^{\prime}(Y)$. Hence $\mathfrak{U}^{\prime}(Y)=\{0\} \oplus \mathfrak{U}(Z)$ with respect to the decomposition $X \oplus Z$ of $B$, where $X=X_{1} \oplus X_{2}$. Hence $\mathfrak{A}(Z)=\mathfrak{A}^{\prime}(Z)$ and so $Z$ is well-embedded in $B$ itself. (Put another way, $\mathfrak{A}^{\prime}(Y) \mid Y=$ $\mathfrak{U}^{\prime}(Z, Y)$, hence $\mathfrak{U}^{\prime}(Z, B)=\mathfrak{U}^{\prime}(Y, B)$ has dimension $(n-2)(n-3) / 2$.) Since $n \geq 4$ is assumed, $\operatorname{dim} Z \geq 2$, so in fact $B$ has a well-embedded 2-dimensional Hilbert subspace as well. Thus we may choose an orthonormal basis $\left(e_{1}, e_{2}, \ldots, e_{n}\right)$ for $B$ so that $\left[e_{1}, e_{2}\right]$ is a well-embedded Hilbert subspace. Let $T_{0} \in \mathfrak{A}(B)$ with $\left[e_{1}, e_{2}\right]=$ range $T_{0}$ (so $\left[e_{3}, \ldots, e_{n}\right]=$ $\left.\operatorname{ker} T_{0}\right)$. Let $Y=\left(e_{2}+e_{3}\right)^{\perp}$. Let $Z$ be a well-embedded Hilbert subspace of $Y$ of dimension $n-2$. Evidently $e_{1} \in Z$. Hence if $Y^{\prime}=Z+\left[e_{2}\right], Y^{\prime}$ is of dimension $n-1$ and $T_{0} \in \mathfrak{U}^{\prime}\left(Y^{\prime}, B\right), T_{0} \notin \mathfrak{U}^{\prime}(Z, B)$. But since

$$
\frac{(n-2)(n-3)}{2}=\operatorname{dim} \mathfrak{A}(Z)=\operatorname{dim} \mathfrak{X}^{\prime}(Z, B)
$$

we obtain

$$
\operatorname{dim} \mathfrak{U}^{\prime}\left(Y^{\prime}\right)=\operatorname{dim} \mathfrak{U}^{\prime}\left(Y^{\prime}, B\right)>\frac{(n-2)(n-3)}{2},
$$


a contradiction. (Note that in fact this argument is "constructive;" the last part actually produces the desired $n$-1-dimensional well-embedded Hilbert subspace as either $Y^{\prime}$ or $Y=\left(e_{2}+e_{3}\right)^{\perp}$.)

We now begin the proof of Theorem 2.2. The following simple result proves useful.

Lemma 2.7. Let $X$ be a finite-dimensional subspace of $B$ and let $\mathfrak{F}_{X}=\left\{B^{\prime}: B^{\prime}\right.$ is a finite-dimensional subspace of $B$ with $\left.X \subset B^{\prime}\right\}$.

(a) Suppose $X$ is orthogonally complemented in all $B^{\prime}$ in $\mathfrak{F}_{X}$. Then $X$ is orthogonally complemented in $B$.

(b) Suppose $X$ is Euclidean and well-embedded in all $B^{\prime}$ in $\mathfrak{F}_{X}$. Then $X$ is well-embedded in $B$.

Proof. Assume $B$ is infinite-dimensional. For each $B^{\prime}$ in $\mathfrak{F}_{X}$, let $\mathcal{O}\left(X \mid B^{\prime}\right)$ denote the orthogonal complement of $X$ in $B^{\prime}$. Then by uniqueness of orthogonal complements, we have that if $B_{1} \subset B_{2}$ belong to $\mathfrak{F}_{X}$, $\mathcal{O}\left(X \mid B_{1}\right)=\mathcal{O}\left(X \mid B_{2}\right) \cap B_{1}$. Now define $Z$ by

$$
Z=\bigcup\left\{\mathcal{O}\left(X \mid B^{\prime}\right): B^{\prime} \in \mathfrak{F}_{X}\right\} \text {. }
$$

It follows that $Z$ is a linear submanifold of $B$ with $X \cap Z=\{0\}$ and $X+Z$ dense in $B$. But in fact $X+Z=B$, for if $b \in B$, then $b \in X+$ $\mathcal{O}(X \mid X+[b])$. If $x \in X$ and $z \in Z$, we have that $\|x+\alpha z\|=\|x+z\|$ for any scalar $\alpha$ with $|\alpha|=1$. But then the same is true for $z \in \bar{Z}$ and $x \in X$. Hence $Z=\bar{Z}$ is the orthogonal complement of $X$. Part (b) of Lemma 2.7 now follows immediately upon using criterion (2) of Proposition 1.11.

We are now prepared for the proof of Theorem 2.2. The complex case is proved in [6], so we restrict to the real case. (Actually the complex case follows, with some elementary reasoning, from the real case.) We also note the following simple consequence of Proposition 1.8: If every finite-dimensional subspace of $X$ is a well-embedded Hilbert subspace, so is $X$.

Indeed, if $Z=\bigcap\left\{\mathcal{O}\left(X^{\prime}\right): X^{\prime}\right.$ is a finite-dimensional subspace of $\left.X\right\}$, then $Z=\mathcal{O}(X) ; X$ is Euclidean by the parallelogram identity and hence well-embedded by Proposition 1.11. To prove Theorem 2.2, by Lemma 2.7 and the above it suffices to prove the special case of finite-dimensional $B$. Thus, fix $B$ finite-dimensional and assume $X$ is a subspace with every one-dimensional subspace of $X$ orthogonally complemented in $B$. We may then assume $B=\left(\mathbf{R}^{n},\|\cdot\|\right)$ with $\|\cdot\|_{E_{n}}$ compatible. Suppose $\operatorname{dim} X$ $=k \geq 2$. Let us first show that every 2-dimensional subspace of $X$ is well-embedded. Let $X_{0}$ be a 2-dimensional subspace; for each $x \in X_{0}$ with $\|x\|=1$, let $P_{x}$ be the orthogonal projection onto $[x]$ and set 
$U_{x}=2 P_{x}-1$. Then $U_{x} \in \mathscr{I}(B)$ and $U_{x} X_{0} \subset X_{0}$. Thus $U_{x} \in G^{\prime}=\{V \in$ $\left.\mathscr{I}(B): V\left|X_{0}^{\perp}=I\right| X_{0}^{\perp}\right\}$. (Here $X_{0}^{\perp}$ is the orthogonal complement of $X_{0}$.) Thus $G^{\prime}$ is an infinite closed group, since $U_{x} \neq U_{x^{\prime}}$ if $x \neq x^{\prime}$. In fact, $G^{\prime} \mid X_{0}$ is an infinite closed subgroup of $\mathscr{I}\left(X_{0},\|\cdot\|_{E_{n}}\right)$ whence $G^{\prime} \mid X_{0} \supset \mathscr{I}_{0}$ where $\mathscr{I}_{0}$ denotes the component of the identity of $\mathscr{I}\left(X_{0},\|\cdot\|_{E_{n}}\right)$. It follows that there is a nonzero $T$ in the tangent space to $G^{\prime}$ at $I$, and of course this $T$ is rank-two Hermitian with range $X_{0}$, so $X_{0}$ is a well-embedded Hilbert subspace. (Of course this argument is quite elementary; no general ideas from Lie groups are really used.) Let $\left\{e_{1}, \ldots, e_{n}\right\}$ be an orthonormal basis for $E_{n}$ so that $X=\left[e_{1}, \ldots, e_{k}\right]$. For each $1 \leq i \neq j \leq k$, let $R_{i j}$ be the operator such that $R_{i j} e_{i}=e_{j}, R_{i j} e_{j}=-e_{i}$ and $R_{i j} e_{l}=0$ for all $l \neq i$ or $j$. Then $R_{i j} \in \mathfrak{U}^{\prime}(X)$, hence

$$
\operatorname{dim} \mathfrak{U}^{\prime}(X) \geq \frac{k(k-1)}{2}=\operatorname{dim}\left[R_{i j}: 1 \leq i<j \leq k\right]
$$

(cf. (22) of [10]). Thus $X$ is well-embedded by Corollary 2.5 .

\section{Orthogonality of Hilbert components.}

We now present the proof of Theorem 2.3. The main step is given by the following result:

LEMMA 2.8. Let $B$ be a real Banach space, $X$ a nonzero well-embedded Hilbert subspace of $B$, and $T$ a rank-two member of $\mathfrak{A}(B)$. Then either $T B \subset X, T B \subset \mathcal{O}(X)$, or there exists a well-embedded Hilbert subspace $Y$ with $X \subsetneq Y$.

Proof. Suppose the first two possibilities don't occur. We must exhibit $\boldsymbol{Y}$

Let $Z=\mathcal{O}(X)$ and $P$ be the natural orthogonal projection onto $X$; that is, $X=P B, Z=\operatorname{ker} P=Q B$ where $Q=I-P$.

Suppose first $X$ is one-dimensional. If $X \subset T B$, the result is trivial, so suppose $X \not \subset T B$. We now claim that $Y=T B+X$ is a well-embedded three-dimensional Hilbert space. Let $\|\cdot\|_{H}$ be a compatible norm on $Y$ and $\left\{e_{1}, e_{2}, e_{3}\right\}$ an orthonormal basis for $Y$ with respect to $\|\cdot\|_{H}$, with $T B=\left[e_{1}, e_{2}\right]$. Thus $T e_{3}=0$. Let $x_{0}$ be a nonzero element of $X$; letting $V=\mathcal{O}(X)$, define $U$ on $B$ by $U(x+v)=x-v$ for $x \in X$ and $v \in V$. Thus $U \in \mathscr{I}(B)$, hence $U T U \in \mathfrak{A}(B)$. Now if $Z_{0}=V \cap Y$, then $Z_{0}$ is orthogonal to $X$ relative to $Y$ and we have $Y=X \oplus Z_{0}$. It follows that $Y$ is invariant under $U$ and since $T B \subset Y$, we have that $U T U(B)=U T(B)$ $\subset Y$. Evidently $T \mid Y$ and $U T U \mid Y$ both belong to $\mathfrak{A}^{\prime}(Y)$. We claim these operators are linearly independent. To see this, we merely need to see that 
$U T U e_{3} \neq 0$. Since $T e_{3}=0$, the linear independence follows. Let $U e_{3}=$ $a e_{1}+b e_{2}+c e_{3}$ for some scalars $a, b$ and $c$. We claim that $a e_{1}+b e_{2} \neq 0$. Were this false, we would have $U e_{3}=c e_{3}$. Now the only eigenvalues of $U \mid Y$ are \pm 1 , and $X$ equals the eigenspace for $+1, Z_{0}$ the eigenspace for -1 . Were $c=1, e_{3} \in X$, but we assumed that $X \not \subset \mathcal{O}(T B)$. Were $c=-1$, then $e_{3} \in Z_{0}$, but since $x_{0}$ is in the orthogonal complement of $Z_{0}, x_{0}$ is in the orthgonal complement of $\left[e_{3}\right]$; i.e., $x_{0}$ is in $\left[e_{1}, e_{2}\right]=\operatorname{range} T$, a contradiction. Thus $T U e_{3}=T\left(a e_{1}+b e_{2}\right) \neq 0$, so $U T U e_{3} \neq 0$. Hence since $\operatorname{dim} \mathfrak{A}^{\prime}(Y) \geq 2, Y$ is a 3-dimensional well-embedded Hilbert space by Theorem 2.1 .

We now assume $\operatorname{dim} X \geq 2$ and set

$$
Z_{0}=Q T B
$$

Evidently we have that $1 \leq \operatorname{dim} Z_{0} \leq 2$.

Case 1. $\operatorname{Dim} Z_{0}=1$.

We shall prove that $Y=X \oplus Z_{0}$ is a well-embedded Hilbert space. Choose $x$ in $X$ and $z$ nonzero in $Z_{0}$ so that $w=z+x$ is in the range of $T$. Then if $w^{\prime}$ is in the range of $T, w^{\prime}=a z+x^{\prime}$ for some scalar $a$ and $x^{\prime}$ in $X$. It follows that we may choose a nonzero $x^{\prime}$ in the range of $T$ with $x^{\prime}$ in $X$. Thus, $T B=\left[x^{\prime}, w\right]$. Suppose now that $x^{\prime}$ and $x$ are linearly independent. Then choose $S \in \mathfrak{A}(B)$ with $\left[x^{\prime}, x\right]=$ range $S$, which is possible since $X$ is well-embedded. It follows that $S$ and $T$ are linearly independent members of $\mathfrak{U}^{\prime}\left(\left[x, x^{\prime}, z\right], B\right)$, whence $\left[x, x^{\prime}, z\right]$ is well-embedded; hence there is a $T^{\prime} \in \mathfrak{A}(B)$ of rank two with $T^{\prime} B=\left[x^{\prime}, z\right]$. If $x$ and $x^{\prime}$ are dependent, this is also true, for then $T$ works. Now choose $v$ in $X$ with $x^{\prime}$ and $v$ independent (possible since $\operatorname{dim} X \geq 2$ ). Choose $S \in$ $\mathfrak{A}(B)$ with $\left[x^{\prime}, v\right]=S(B)$; we again obtain that $\left[x^{\prime}, v, z\right]$ is well-embedded since $S$ and $T^{\prime}$ are linearly independent members of $\mathfrak{A}^{\prime}\left(\left[x^{\prime}, v, x\right], B\right)$. But obviously any one-dimensional subspace of $Y$ is a subspace of $\left[x^{\prime}, v, z\right]$ for some choice of $v$, so by Theorem 2.2, $Y$ is well-embedded.

\section{Case 2. $\operatorname{dim} Z_{0}=2$.}

Choose $z, z^{\prime}$ linearly independent members of $Z_{0}$ and $x, x^{\prime}$ in $X$ so that $x \neq 0$ and $T B=\left[w, w^{\prime}\right]$ where $w=x+z$ and $w^{\prime}=x^{\prime}+z^{\prime}$. (We cannot have both $x$ and $x^{\prime}$ zero, for then $T B \subset \mathcal{O}(X)$ contradicting our assumptions.) Then $[x]$ is orthogonally complemented in $B$ since $X$ is a well-embedded Hilbert subspace. It follows that since $z \in \mathcal{O}([x]), w=x$ $+z \notin \mathcal{O}([x])$; hence $T B \not \subset \mathcal{O}([x])$. Thus by our proof for the case " $\operatorname{dim} X$ $=1$," we obtain that $\left[w, w^{\prime}, x\right]$ is a well-embedded Hilbert subspace. 
Hence there is a $T^{\prime}$ in $\mathfrak{A}(B)$ with $T^{\prime}(B)=[w, x]=[x, z]$. Thus $\operatorname{dim} Q T^{\prime} B$ $=1$ so we have reduced to case 1 and the proof is complete.

We are now prepared for the proof of Theorem 2.3. Let $H_{1}$ and $H_{2}$ be as in its statement. It suffices to show that $H_{2} \subset \mathcal{O}\left(H_{1}\right)$. Our work so far shows that $\mathcal{O}\left(H_{1}\right)=\bigcap\left\{\operatorname{ker} T: T \in \mathfrak{A}(B), T\right.$ is rank 2 and $\left.T B \subset H_{1}\right\}$. (See the third remark following the statement of Theorem 2.2 above.) Thus if $\mathrm{H}_{2} \not \subset \mathcal{O}\left(H_{1}\right)$, we could choose a rank-2 skew-Hermitian operator $T$ on $B$ with $T B \subset H_{1}$ yet $H_{2} \not \subset \operatorname{ker} T$. This shows $T B \not \subset \mathcal{O}\left(H_{2}\right)$. Indeed, $T B \subset \mathcal{O}\left(H_{2}\right)$ implies $\mathcal{O}(T B) \supset H_{2}$ but $\mathcal{O}(T B)=\operatorname{ker} T$. Hence by Lemma 2.8, $T B \subset \mathrm{H}_{2}$. Of course this shows $\mathrm{H}_{1} \cap \mathrm{H}_{2}$ is nonempty (in fact $\operatorname{dim} H_{1} \cap H_{2} \geq 2$ ). Now choose $x$ nonzero in $H_{1} \cap H_{2}$ and $y \in H_{2} \sim H_{1}$. (There must be such a $y$ since $H_{1}$ and $H_{2}$ are distinct, so $H_{2} \not \subset H_{1}$.) Choose $S \in \mathfrak{A}(B)$ with $[x, y]=$ range $S$ (possible since $[x, y]$ is well-embedded in $H_{2}$ and hence in $B$ ). Now evidently $S B \not \subset H_{1}$. But in fact $S$ is invertible on $[x, y]$, hence $S x \neq 0$, so $H_{1} \not \subset \operatorname{ker} S$, that is $S B \not \subset \mathcal{O}\left(H_{1}\right)$. Thus by Lemma 2.8, $H_{1}$ could not be a Hilbert component. This contradiction completes the proof of 2.3 .

\section{Lie-classification of spaces of low dimension.}

We conclude this section with some remarks concerning the Lie-classification problem. Theorem 2.1 and standard results about Lie groups lead easily to the Lie-classification of real Banach spaces of dimension up to four. Indeed, the only case not covered by Theorem 2.1 is where $B$ is four-dimensional and $\operatorname{dim} \mathfrak{A}(B)=2$. We then claim that there is a complex two-dimensional non-Euclidean space $Z$ with a normalized oneunconditional basis $\left(u_{1}, u_{2}\right)$ so that $B$ is isometric to $Z_{\mathbf{R}}$ (where $Z_{\mathbf{R}}$ denotes $Z$ regarded as a real Banach space). Assuming $B=\left(\mathbf{R}^{4},\|\cdot\|\right)$ with $\|\cdot\|_{E_{4}}$ and $\|\cdot\|$ compatible, we obtain that $\mathfrak{A}(B)$ is orthogonally equivalent to $s \mathrm{O}_{2} \oplus s \mathrm{O}_{2}$. To prove the claim, let $B$ have the above representation and let $G$ denote the component of the identity of $\mathscr{I}(B)$. Then $G$ is a two-dimensional compact connected Lie group. By the known structure of such objects, $G$ must be commutative and in fact isomorphic to $\mathrm{SO}_{2} \times \mathrm{SO}_{2}=\mathrm{T}^{2}$, the two-dimensional torus. Moreover the isomorphism from $T^{2}$ onto $G$ is then a representation of $T^{2}$ in $S_{4}$ and hence must be reducible. We thus in fact obtain two-dimensional orthogonal subspaces $X$ and $Y$ with $G=G|X \oplus G| Y$. It follows that both $X$ and $Y$ are Euclidean and in fact $\mathscr{I}(B)=\mathscr{I}(X) \oplus \mathscr{I}(Y)$. Thus, $X$ and $Y$ are each well-embedded; it now follows easily that $B$ has the stated structure, since we obtain that $B$ is a functional unconditional sum of $X$ and $Y$. Moreover we have that $\mathfrak{A}(B)=\mathfrak{A}(X) \oplus \mathfrak{A}(Y)$ and of course $\mathfrak{A}(X)$ and $\mathfrak{A}(Y)$ are both orthogonally equivalent to $\mathrm{SO}_{2}$. 
In fact, we see that such a $B$ gives the simplest possible example of a non-Euclidean $F H S$, for $X$ and $Y$ are the Hilbert components of $B$. It is moreover easily seen that the basis $\left(u_{1}, u_{2}\right)$ for the corresponding complex space $Z$ is essentially unique. Indeed, we shall in fact show in the next section that if $U$ is an isometry of $Z$, there exist complex numbers $\alpha_{i}$ of modulus 1 and a permutation $\sigma$ of $(1,2)$ so that $U\left(u_{i}\right)=\alpha_{i} u_{\sigma(i)}$. (This also follows immediately from the results in [6].)

It is possible to give an elementary proof of the above structural result, without passing through the theory of Lie groups. Here is the argument.

It is easily seen that if $A$ is a nonzero $2 \times 2$ matrix, then

$$
A \text { and } R_{0} A \text { are linearly independent. }
$$

Indeed, otherwise $R_{0} A=c A$ for some $c$ implies $R_{0}^{2} A=-A=c R_{0} A=$ $c^{2} A$ so $c^{2}=-1$, a contradiction.

Now assume $\mathfrak{A}(B)$ is two-dimensional. Once it is proved that $\mathfrak{A}(B)$ has a rank-two element, we may choose an orthonormal basis so that

$$
G=\left[\begin{array}{cc}
R_{0} & 0 \\
0 & 0
\end{array}\right]
$$

is (the matrix of) a member of $\mathfrak{A}$ (with respect to this basis). It follows that we may choose a second nonzero element $T$ in $\mathfrak{U}(B)$ with

$$
T=\left[\begin{array}{cc}
0 & A \\
-A^{*} & \alpha R_{0}
\end{array}\right]
$$

for some real $\alpha$. Then

$$
[G, T]=\left[\begin{array}{cc}
0 & R_{0} A \\
A^{*} R_{0} & 0
\end{array}\right]
$$

belongs to $\mathfrak{A}(B)$ : were $A$ nonzero, $G, T$ and $[G, T]$ would be linearly independent by (20), contradicting $\operatorname{dim} \mathfrak{A}(B)=2$. Thus $A=0, \alpha \neq 0$ and we have proved in fact $\mathfrak{U}(B)=s \mathfrak{O}_{2} \oplus s \mathfrak{O}_{2}$, which easily implies the existence of the desired complex $Z$.

Now suppose $\mathfrak{A}(B)$ has no rank-2 elements. We then reason to a contradiction as follows. Again, we may choose one element

$$
G=\left[\begin{array}{cc}
R_{0} & 0 \\
0 & c R_{0}
\end{array}\right]
$$

in $\mathfrak{A}(B)$ with $c>0$ say (with respect to a suitable basis). Next it follows there is no element $T$ in $\mathfrak{A}$ with

$$
T=\left[\begin{array}{cc}
0 & A \\
-A^{*} & R_{0}
\end{array}\right]
$$


For then $A \neq 0$, and again

$$
[G, T]=\left[\begin{array}{cc}
0 & -R_{0} A \\
A^{*} R_{0} & 0
\end{array}\right]
$$

implies $G, T$ and $[G, T]$ are linearly independent, by (21). It then follows there is a second nonzero element $T$ in $\mathfrak{A}$ with

$$
T=\left[\begin{array}{cc}
0 & A \\
-A^{*} & 0
\end{array}\right] \text {. }
$$

Then also $A$ is invertible, since $T$ cannot be rank-2. We now obtain

$$
[G, T]=\left[\begin{array}{cc}
0 & R_{0} A-c A R_{0} \\
-\left(T_{0} A-c A R_{0}\right) * & 0
\end{array}\right]
$$

Hence there must be a real number $t$ so that

$$
R_{0} A-c A R_{0}=t A
$$

(or else $\operatorname{dim} \mathfrak{U}(B) \geq 3$ ). This implies $A^{-1} R_{0} A=c R_{0}+t I$ so

$$
-I=\left(A^{-1} R_{0} A\right)^{2}=\left(-c^{2}+t^{2}\right) I+2 t c R_{0} .
$$

Now (23) implies $t=0$ and $c=1$ (since $c>0$ was assumed). Thus $[G, T]=0$; that is, $G$ and $T$ commute. It follows there is an orthogonal matrix $U$ so that $U^{*} G U=G$ and

$$
U^{*} T U=\left[\begin{array}{cc}
a R_{0} & 0 \\
0 & b R_{0}
\end{array}\right]
$$

for some real numbers $a$ and $b$. (This is the real-version of the fact that commuting normal complex matrices can be simultaneously unitarily diagonalized.) Since $T \neq G, a \neq b$, so in fact we deduce

$$
\left[\begin{array}{cc}
R_{0} & 0 \\
0 & 0
\end{array}\right]
$$

is in $\mathfrak{A}$ after all, a contradiction.

REMARK. The following result follows from complexification and a result of Vidav [14];1 alternatively, it would appear to follow from the natural "Lie-group" generalization of the above argument:

Let $B$ be a real $2 n$-dimensional Banach space. Then $B$ is isometric to $X_{\mathbf{R}}$ for some complex $n$-dimensional space $X$ with a one unconditional basis if and only if $\mathfrak{A}(B)$ has a commutative n-dimensional subspace.

3. The isometric structure of FHS. Many of our results here follow in a straightforward way from the machinery developed in the first two sections. We organize the presentation as follows: we first prove Theorem 
1 of the introduction, and also give the structure of the span of the Hilbert components of a general Banach space. This leads to a natural splitting of arbitrary one-unconditional bases with one part spanning the "FHS-part" of the space. We next give the detailed isometric classification of FHS spaces of arbitrary cardinality, as well as the characterizations of their groups of isometries and Lie algebras. We proceed then to give some complementation-permanence properties of FHS spaces and general spaces with a one-unconditional basis. Unless explicitly stated otherwise, we shall assume that $B$ is a real nonzero Banach space. (Later on, we discuss the relationship with our work and the previously known analogues for complex Banach spaces.)

The Functional Hilbertian part of a Banach space and the structure of spaces with a one-unconditional basis.

We first introduce some notation. Let $\mathscr{H}=\mathscr{H}(B)$ denote the set of Hilbert components $H$ of $B$ with $\operatorname{dim} H \geq 2$. (For any Banach space $X$, $\operatorname{dim} X$ denotes the least cardinal corresponding to a subset of $X \sim\{0\}$ with linear span dense in $X$.) When we set $\mathscr{H}=\left\{H_{\lambda}\right\}_{\lambda \in \Gamma}$ we shall assume that $H_{\lambda} \neq H_{\lambda^{\prime}}$ if $\lambda \neq \lambda^{\prime}$; i.e., this is simply a "distinct" indexing of $\mathscr{H}$. We set $\mathrm{FH}(B)=[H: H \in \mathscr{H}]$ and call $\mathrm{FH}(B)$ the Functional Hilbertian part of $B$. We set $\operatorname{Or}(B)=\{x:[x]$ is a Hilbert component of $B$ or $x=0\}$.

TheOREM 3.1. Let $X=\mathrm{FH}(B)$. Then $\mathscr{H}$ is a functional unconditional decomposition of $X . X=[T B: T \in \mathfrak{A}(B)$ with $\operatorname{rank} T=2]$. [x] is orthogonal to $H$ for all $H$ in $\mathscr{H}$ and $x$ in $\operatorname{Or}(B)$. If $X$ is orthogonally complemented in $B$, then $\mathcal{O}(X)=\cap\{\mathcal{O}(H): H \in \mathscr{H}\} . X$ is orthogonally complemented in $B$ if either of the following occur:

(a) $c_{0}$ does not embed in $X$

or

(b) $B=[x:[x]$ is orthogonally complemented in $B]$.

RemarKs. (1) Notice that if $B$ has a one-unconditional basis, then (b) holds so $X=\mathrm{FH}(B)$ is orthogonally complemented. Moreover if (b) holds, then $\mathcal{O}(X)=[\mathcal{O} r(B)]$. Indeed, if $[x]$ is orthogonally complemented in $B$, then either $x \in \operatorname{Or}(B)$ or $x \in \mathrm{FH}(B)$ by the existence of Hilbert components. If (b) holds, then Theorem 3.1 yields that $\operatorname{Or}(B) \subset \mathcal{O}(X)$.

(2) Suppose $\left\{H_{\lambda}: \lambda \in \Gamma\right\}=\mathscr{H}(B)$. Then by Proposition 1.7 and Theorem 3.1, we have that $\mathrm{FH}(B)$ is a functional unconditional sum of $\left\{H_{\lambda}: \lambda \in \Gamma\right\}$ and hence we obtain Theorem 1 of the Introduction (the implication (a) $\Rightarrow$ (b) of Theorem 1 is trivial). 
Proof of Theorem 3.1. Let $n \geq 1$ and $H_{1}, \ldots, H_{n}$ be distinct members of $\mathscr{H}$. By Theorem 2.3, we have that the $H_{i}$ 's are orthogonal; that is, $H_{i} \subset \mathcal{O}\left(H_{j}\right)$ for all $i \neq j$. Let $x_{1}, x^{\prime}, \ldots, x_{n}, x_{n}^{\prime}$ be given with $\left\|x_{i}\right\|=\left\|x_{i}^{\prime}\right\|$ and $x_{i}, x_{i}^{\prime} \in H_{i}$ for all $i$. For each $i$, since $H_{i}$ is well-embedded, we may choose $U_{i} \in \mathscr{I}(B)$ with $U_{i}(y)=y$ for all $y \in \mathcal{O}\left(H_{i}\right)$ and $U_{i} x_{i}=x_{i}^{\prime}$. Let $U=U_{1} \cdot U_{2} \cdot \ldots \cdot U_{n}$. Then evidently $U \in \mathscr{I}(B)$ and $U\left(\sum x_{i}\right)=\sum x_{i}^{\prime}$. Hence $\left\|\sum x_{i}^{\prime}\right\|=\left\|\sum x_{i}\right\|$, proving that $\mathscr{H}$ is a functional unconditional decomposition of $X$. If $x \in H$ for some $H \in \mathscr{H}$, since $\operatorname{dim} H \geq 2$, there is a $T \in \mathfrak{A}(B)$ with rank $T=2$ and $x \in T B$. If conversely $T \in \mathfrak{A}(B)$ with rank $T=2, T B$ is a well-embedded 2-dimensional Hilbert subspace of $B$, hence by Theorem 1.12 , there is an $H \in \mathscr{H}$ with $T B \subset H$. The second assertion of 3.1 is thus established; the third one follows immediately from Theorem 2.3. The fourth assertion and the first part of the fifth one follow immediately from the preceding parts of 3.1 and Corollary 1.9. For the final assertion, let $Y=\bigcap\{\mathcal{O}(H): H \in \mathscr{H}\}$. If $Z=X \oplus Y$, then $X$ is orthogonally complemented in $Z$ with $Y$ the orthogonal complement, as noted in our proof of Proposition 1.8. Now $\operatorname{Or}(B) \subset Y$ while if $[x]$ is orthogonally complemented in $B$ and $x \notin \mathcal{O} r(B)$, then there is an $H \in \mathscr{H}$ with $x \in H$ and so $x \in X$. Hence if (b) of 3.1 holds, $Z=B$.

Our next result leads to a description of the group of isometries of an FHS space as well as a simple characterization of its Lie algebra. It is again a natural analogue of a known result for complex spaces [6].

Proposition 3.2. Let $H$ be a Hilbert component of $B, T \in \mathfrak{U}(B)$ and $U \in \mathscr{I}(B)$. Then $U H$ is a Hilbert component. If $\operatorname{dim} H>1, T H \subset H$.

Proof. The first assertion is evident. To see the second one, let $b \in H$. We first show there is a $\delta>0$ so that $e^{x T} b \in H$ for all $x$ with $|x|<\delta$. Assume $\|b\|=1$ for convenience and define $f$ by $f(x)=\left\|b-e^{x T} b\right\|$. Evidently $f$ is continuous. Now by the first assertion and Theorem 2.3, if $e^{x T} b \notin H$, then $e^{x T} b \in \mathcal{O}(H)$, whence $f(x) \geq\|b\|=1$. Since $\lim _{x \rightarrow 0} f(x)=0$, the existence of $\delta$ follows. But then it follows that $d\left(e^{x T} b\right) / d x \in H$ for $|x|<\delta$; of course this equals $T e^{x T} b$, whence setting $x=0, T b \in H$.

REMARKS. (1) We do not know if this assertion remains true if one assumes $\operatorname{dim} H=1$. Notice the assertion is then equivalent to: $H \subset \operatorname{ker} T$.

(2) Actually, if $\operatorname{dim} H>1, H$ is a Hilbert component, and $\mathscr{I}_{0}(B)$ is the component of the identity of $\mathscr{I}(B)$, then $U H \subset H$ for all $U \in \mathscr{I}_{0}(B)$ (so in particular $e^{x T} H \subset H$ for all real $x$ and $T \in \mathfrak{A}(B)$ ). (We work with 
the norm-topology on $\mathscr{I}(B)$.) Indeed, let $G=\left\{U \in \mathscr{I}_{0}(B): U H \subset H\right\}$. $G$ is evidently a closed subgroup of $\mathscr{I}_{0}(B)$. To see that $G$ is open, fix $b \in H$ with $\|b\|=1$, let $U \in G$ and $V \in \mathscr{I}_{0}(B)$ with $\|V-U\|<1$. Then were $V b \notin H, V b \in \mathcal{O} H$; hence $\|(V-U)(b)\| \geq\|U b\|=1$. But $\|(V-U)(b)\|$ $<1$; hence $V \in G$, so $G=\mathscr{I}_{0}(B)$.

Corollary 3.3. Let $X=F H(B)$ and $Y=\bigcap\{\mathcal{O}(H): H \in \mathscr{H}\}$. Then $X$ and $Y$ are both invariant under $\mathscr{I}(B)$ and $\mathfrak{A}(B)$. Moreover if $U \in \mathscr{I}(B)$, then $\mathscr{H}=\{U H: H \in \mathscr{H}\}$.

Proof. Let $U \in \mathscr{I}(B)$ and $T \in \mathfrak{A}(B)$. If $H \in \mathscr{H}$, then $U H \in \mathscr{H}$ since $\operatorname{dim} U H=\operatorname{dim} H \geq 2$ and $U H$ is a Hilbert component. Thus evidently $U X \subset X$. Hence also incidentally $U^{-1} X \subset X$, which shows that $U X=X$. Notice this also shows in fact $\mathscr{H}=\{U H: H \in \mathscr{H}\}$. Now suppose $y \in Y$. Then since $U$ is a surjective isometry, we have that $U y \in \mathcal{O}(U H)$ for any $H \in \mathscr{H}$; hence again $U y \in Y$. (This also shows incidentally that $U Y=Y$.) Now let $T \in \mathfrak{A}(B)$ and $y \in Y$. Then $e^{x T} y \in Y$ for all real $x$. Thus $d\left(e^{x T} y\right) /\left.d x\right|_{x=0}=T y$ belongs to $Y$, since $Y$ is closed. It follows immediately from Proposition 3.2 that $T X \subset X$.

ReMARK. Suppose $X=F H(B)$ is orthogonally complemented and $Y=\mathscr{O}(X)$. We obtain that $\mathscr{I}(B)=\mathscr{I}(X) \oplus \mathscr{I}(Y)$ precisely when $X$ and $Y$ are well-embedded in $B$. Then also $\mathfrak{A}(B)=\mathfrak{A}(X) \oplus \mathfrak{A}(Y)$. In particular, this occurs if $B$ is a functional unconditional sum of $X$ and $Y$. (We prove later that in any case, $\mathfrak{A}(B)=\mathfrak{A}(X) \oplus \mathfrak{A}^{\prime}(Y)$.)

Recall from the Introduction that if $U$ is a real Banach space with normalized one-unconditional basis $\left(u_{\gamma}\right)_{\gamma \in \Gamma}$, then $U$ is said to be pure if there are no rank-two skew-Hermitian operators on $U$. Otherwise, $U$ is called impure. We show in Theorem 3.6 that if $U$ is pure, $U$ has a trivial Lie algebra. Our next result gives on the other hand a useful criterion testing the impurity of a space $U$.

CoROllaRy 3.4. Let $\left(u_{\gamma}\right)_{\gamma \in \Gamma}$ be a normalized one-unconditional basis for $U$. Then $U$ is impure if and only if there exist $\alpha \neq \beta$ in $\Gamma$ so that $\left(u_{\alpha}, u_{\beta}\right)$ is isometrically equivalent to the usual basis for Euclidean 2-dimensional space and for all $x$ and $x^{\prime}$ in $\left[u_{\alpha}, u_{\beta}\right]$ with $\|x\|=\left\|x^{\prime}\right\|$ and all $y \in\left[\left\{u_{\gamma}: \gamma \neq \alpha\right.\right.$ or $\left.\left.\beta\right\}\right],\|x+y\|=\left\|x^{\prime}+y\right\|$.

Proof. If there exist such $\alpha$ and $\beta,\left[u_{\alpha}, u_{\beta}\right]$ is a well-embedded Hilbert subspace of $U$ and hence the range of a rank-two skew-Hermitian operator on $U$. Suppose that $U$ is impure. Then there exists an $H \in \mathscr{H}(U)$; 
that is, $H$ is a Hilbert component with $\operatorname{dim} H \geq 2$. If $\alpha \in \Gamma$, either $u_{\alpha} \in \mathcal{O} r(U)$ in which case $\left[u_{\alpha}\right]$ is orthogonal to $H$ by Theorem 3.1, or there exists an $H^{\prime}$ in $\mathscr{H}$ with $u_{\alpha}$ in $H^{\prime}$ (since $\left[u_{\alpha}\right]$ is an orthogonally complemented subspace). If $H^{\prime}=H, u_{\alpha} \in H$, otherwise [ $\left.u_{\alpha}\right]$ is again orthogonal to $H$. It follows that there exist $\alpha \neq \beta$ in $\Gamma$ with $u_{\alpha}, u_{\beta}$ both in $H$. Otherwise $\mathcal{O}(H)=U$ or $\mathcal{O}(H)$ is of codimension one in $U$, both of which are absurd. Then $\left[u_{\alpha}, u_{\beta}\right]$ is a well-embedded Hilbert subspace of $U$. Since $\mathcal{O}\left(\left[u_{\alpha}, u_{\beta}\right]\right)=\left[\left\{u_{\gamma}: \gamma \neq \alpha\right.\right.$ or $\left.\left.\beta, \gamma \in \Gamma\right\}\right], \alpha$ and $\beta$ have the desired properties.

Our argument for Corollary 3.4 easily yields the following result, which in turn leads to a description of unconditional bases for FHS spaces:

LEMMA 3.5. Let $U$ have a normalized one-unconditional basis $\left(u_{\alpha}\right)_{\alpha \in \Gamma}$ and let $H \in \mathscr{H}(U)$. Then $H=\left[u_{\alpha}: \alpha \in \Gamma\right.$ and $\left.u_{\alpha} \in H\right]$.

Proof. Our argument for Corollary 3.4 showed that for every $\alpha$, either $u_{\alpha} \in H$ or $\left[u_{\alpha}\right]$ is orthogonal to $H$. Hence if $H^{\prime}=\left[u_{\alpha}: \alpha \in \Gamma\right.$ and $\left.u_{\alpha} \in H\right]$, then $\mathcal{O}\left(H^{\prime}\right)=\left[u_{\beta}: u_{\beta} \notin H\right.$ and $\left.\beta \in \Gamma\right] \subset \mathcal{O}(H)$; hence $H^{\prime} \supset H$, so $H^{\prime}=H$.

We may now describe the structure of a one-unconditional basis for a general $B$. The basis splits into two pieces, one spanning $\mathrm{FH}(B)$, the other being the part in $\operatorname{Or}(B)$. The FH-part is naturally partioned by the Hilbert components of $\mathrm{FH}(B)$, i.e., by $\mathscr{H}(B)$. If one selects one basis element out of each member of $\mathscr{H}(B)$ and combines these elements with the $\mathcal{O} r$ part, the result spans a pure space. Here is the precise result.

THEOREM 3.6. Assume $\Lambda$ and $\Gamma$ are disjoint (index) sets. Let $\left(u_{\alpha}\right)_{\alpha \in \Gamma}$ be a normalized one-unconditional basis for $B,\left\{H_{\lambda}: \lambda \in \Lambda\right\}=\mathscr{H}(B)$, $\Gamma_{O_{r}}=\left\{\alpha: u_{\alpha} \in \operatorname{Or}(B)\right\}, \Gamma_{\mathrm{FH}}=\Gamma \sim \Gamma_{O_{r}}$, and for each $\lambda \in \Lambda$, let $\Gamma_{\lambda}=\{\alpha:$ $\left.u_{\alpha} \in H_{\lambda}\right\}$ and $\alpha(\lambda)$ be a chosen element of $\Gamma_{\lambda}$.

(a) $\mathrm{FH}(B)=\left[u_{\alpha}: \alpha \in \Gamma_{\mathrm{FH}}\right]$ so $\mathcal{O}(\mathrm{FH}(B))=\left[u_{\alpha}: \alpha \in \Gamma_{\mathscr{O}_{r}}\right]$.

(b) For each $\lambda \in \Lambda, H_{\lambda}=\left[u_{\alpha}: \alpha \in \Gamma_{\lambda}\right]$. The $\Gamma_{\lambda}$ 's are a partition of $\Gamma_{\mathrm{FH}}$. That is, $\Gamma_{\mathrm{FH}}=U_{\lambda \in \Lambda} \Gamma_{\lambda}$ and $\Gamma_{\lambda} \cap \Gamma_{\lambda^{\prime}}=\varnothing$ if $\lambda \neq \lambda^{\prime}$.

(c) Let $\Gamma^{\prime}=\Lambda \cup \Gamma_{o_{r}}$. Define $v_{\gamma}$ for $\gamma \in \Gamma^{\prime}$ by $v_{\gamma}=u_{\alpha(\gamma)}$ if $\gamma \in \Lambda$ and $v_{\gamma}=u_{\gamma}$ if $\gamma \in \Gamma_{\mathcal{O}_{r}}$; set $V=\left[v_{\gamma}: \gamma \in \Gamma^{\prime}\right]$. Then $V$ is pure.

Proof. Set $X=\left[u_{\alpha}: \alpha \in \Gamma_{\mathrm{FH}}\right]$. It follows by the preceding Lemma and Theorem 3.1 that if $H \in \mathscr{H}(B)$, then $H \subset X$. For $H=\left[u_{\alpha}: u_{\alpha} \in H\right]$ but if $\alpha \in \Gamma_{\mathscr{O}_{r}}, u_{\alpha} \notin H$ since $\left[u_{\alpha}\right]$ is orthogonal to $H$. Hence we obtain 
that $\mathrm{FH}(B) \subset X$. But if $\alpha \in \Gamma_{\mathrm{FH}}$, there must be an $H \in \mathscr{H}(B)$ with $u_{\alpha} \in H$ by the existence of Hilbert components and the fact that $\left[u_{\alpha}\right]$ is not a Hilbert component. Hence $X \subset F H(B)$ so (a) follows. (b) follows immediately from the preceding Lemma and the orthogonality of the $H_{\lambda}$ 's. It remains to prove (c). For convenience, let $H_{\gamma}=\left[v_{\gamma}\right]=\left[u_{\alpha(\gamma)}\right]$ for all $\gamma \in \Gamma_{o_{r}}$. Then we have that $\left\{H_{\gamma}: \gamma \in \Gamma^{\prime}\right\}$ are orthogonal Hilbert components of $B$ and of course $B=\left[H_{\gamma}: \gamma \in \Gamma^{\prime}\right]$. Suppose (c) is false. By Corollary 3.4 we may choose $\alpha \neq \beta$ in $\Gamma^{\prime}$ so that $\left[v_{\alpha}, v_{\beta}\right]$ is Euclidean and well-embedded in $V$. We shall show that $H_{\alpha}+H_{\beta}$ is a well-embedded Hilbert subspace of $B$, contradicting the fact that $H_{\alpha}$ and $H_{\beta}$ are Hilbert components. Let $\bar{\Gamma}=\Gamma^{\prime} \sim\{\alpha, \beta\}$. Let $x, x^{\prime} \in H_{\alpha}$ and $y, y^{\prime} \in H_{\beta}$ with $\|x+y\|=\left\|x^{\prime}+y^{\prime}\right\|$ and let $z \in\left[H_{\gamma}\right]_{\gamma \in \bar{\Gamma}}$. First we have that there is an isometry $U$ of $B$ with $U x=\|x\| v_{\alpha}$ and $U y=\|y\| v_{\beta}$, hence

$$
\|x+y\|=\|\| x\left\|v_{\alpha}+\right\| y\left\|v_{\beta}\right\|=\left(\|x\|^{2}+\|y\|^{2}\right)^{1 / 2},
$$

showing $H_{\alpha}+H_{\beta}$ is Euclidean. Next, we may choose unique $z_{\lambda} \in H_{\lambda}$ for all $\lambda \in \bar{\Gamma}$ so that $z=\sum_{\lambda \in \bar{\Gamma}} z_{\lambda}$ (the series converging unconditionally); then we have that

$$
\|x+y+z\|=\|\| x\left\|v_{\alpha}+\right\| y\left\|v_{\beta}+\sum_{\lambda \in \bar{\Gamma}}\right\| z_{\lambda}\left\|v_{\lambda}\right\|
$$

(since the $H_{\gamma}$ 's are a functional unconditional decomposition of $B$ )

$$
=\|\| x^{\prime}\left\|v_{\alpha}+\right\| y^{\prime}\left\|v_{\beta}+\sum_{\lambda \in \Lambda}\right\| z_{\lambda}\left\|v_{\lambda}\right\|
$$

(since $\left[v_{\alpha}, v_{\beta}\right]$ is well-embedded in $U$ and $\|x+y\|=\left\|x^{\prime}+y^{\prime}\right\|$ )

$$
=\left\|x^{\prime}+y^{\prime}+z\right\| \text {. }
$$

Thus $H_{\alpha}+H_{\beta}$ is well-embedded, a contradiction. This completes the proof of Theorem 3.6.

Our preceding results easily yield a complete isometric description of FHS spaces. To give this, we introduce some notation: suppose $B$ is a functional unconditional sum of $\left(Y_{\alpha}\right)_{\alpha \in \Gamma}$. Then formally, $B \subset \Pi_{\alpha \in \Gamma} Y_{\alpha}$. For each $\alpha \in \Gamma$, let $Y_{\alpha}^{\prime}=\left\{b \in B: b_{\beta}=0\right.$ for all $\left.\beta \neq \alpha\right\}$. We refer to the $Y_{\alpha}^{\prime \prime}$ 's as the canonical images of the $Y_{\alpha}^{\prime}$ 's in $B$. We say that $B$ has an isometrically unique one-unconditional basis if $B$ has a normalized oneunconditional basis $\left(u_{\alpha}\right)_{\alpha \in \Gamma}$ so that if $\left\{v_{\alpha}\right\}_{\alpha \in \Lambda}$ is a normalized one-unconditional basis for $B$, there is a bijection $\sigma: \Gamma \rightarrow \Lambda$ with $\left(v_{\sigma(\alpha)}\right)$ isometrically equivalent to $\left(u_{\alpha}\right)_{\alpha \in \Gamma}$; that is, there is a $T \in \mathscr{I}(B)$ with 
$T u_{\alpha}=v_{\sigma(\alpha)}$ for all $\alpha \in \Gamma$. (Recall that for Hilbert spaces $X$ and $X^{\prime}$, $\operatorname{dim} X=\operatorname{dim} X^{\prime}$ if and only if $X$ is isometric to $X^{\prime}$.)

THEOREM 3.7. Let $B$ be FHS. Then $B$ has an isometrically unique one-unconditional basis. There exists a one-unconditional basis $u=\left(u_{\alpha}\right)_{\alpha \in \Gamma}$ for a pure space $U$ and Hilbert spaces $\left(H_{\alpha}\right)_{\alpha \in \Gamma}$ with $\operatorname{dim} H_{\alpha} \geq 2$ for all $\alpha$ so that $B$ is isometric to $\left(\Sigma_{\Gamma} \oplus H_{\alpha}\right)_{\underline{u}}$. The $H_{\alpha}^{\prime \prime}$ 's are then the Hilbert components of $B$, where the $H_{\alpha}^{\prime \prime}$ s are the canonical images of the $H_{\alpha}$ 's in $B$. The representation is unique in the following sense: if $B$ is isometric to $\left(\Sigma_{\Lambda} \oplus \tilde{H}_{\beta}\right)_{\underline{v}}$ where $\underline{v}=\left(v_{\lambda}\right)_{\lambda \in \Lambda}$ is a normalized one-unconditional basis for some pure space and $\tilde{H}_{\beta}$ are Hilbert spaces for all $\beta$, then there is a bijection $\sigma: \Gamma \rightarrow \Lambda$ with $\left(v_{\sigma(\gamma)}\right)$ isometrically equivalent to $\left(u_{\gamma}\right)$ and $\operatorname{dim} \tilde{H}_{\sigma(\gamma)}=$ $\operatorname{dim} H_{\gamma}$ for all $\gamma \in \Gamma$.

Proof. The argument is a routine application of our preceding results. However the generality of the result renders the notation somewhat cumbersome.

Let $\left(H_{\alpha}\right)_{\alpha \in \Gamma}$ be the Hilbert components of $B$ and for each $\alpha \in \Gamma$, let $u_{\alpha} \in H_{\alpha}$ with $\left\|u_{\alpha}\right\|=1$; set $U=\left[u_{\alpha}\right]_{\alpha \in \Gamma}$ and $\underline{u}=\left(u_{\alpha}\right)_{\alpha \in \Gamma}$. Then by Lemma 3.5, Theorem 3.1 and Theorem 3.6, $B$ is isometric to $\left(\sum_{\alpha \in \Gamma} \oplus H_{\alpha}\right)_{\underline{u}}$ and $U$ is pure. For each $\alpha \in \Gamma$, let $\left(u_{\alpha \beta}\right)_{\beta \in \Gamma_{\alpha}}$ be a complete orthonormal basis for $H_{\alpha}$, where $\Gamma_{\alpha}=\operatorname{dim} H_{\alpha}$. Then setting $\tilde{\Gamma}=U_{\alpha \in \Gamma}\{\boldsymbol{\alpha}\} \times \Gamma_{\alpha},\left(u_{\gamma}\right)_{\gamma \in \tilde{\Gamma}}$ is a normalized one-unconditional basis for $B$. Suppose $\left(v_{\tau}\right)_{\tau \in W}$ is a normalized one-unconditional basis for $B$. For each $\alpha \in \Gamma$, let $W_{\alpha}=\left\{\tau: v_{\tau} \in H_{\alpha}\right\}$. Since Hilbert spaces have isometrically unique unconditional bases, it follows by Theorem 3.6 that there exists a bijection $\sigma_{\alpha}:\{\alpha\} \times \Gamma_{\alpha} \rightarrow W_{\alpha}$ with $\left(v_{\sigma(\alpha \beta)}\right)_{\beta \in \Gamma_{\alpha}}$ isometrically equivalent to $\left(u_{\alpha \beta}\right)_{\beta \in \Gamma_{\alpha}}$. Since $\left\{W_{\alpha}: \alpha \in \Gamma\right\}$ is a disjoint partition of $W$, $\sigma=\bigcup_{\alpha \in \Gamma} \sigma_{\alpha}$ is a bijection from $\tilde{\Gamma}$ onto $W$. To see that $\left(u_{\gamma}\right)_{\gamma \in \Gamma}$ is isometrically equivalent to $\left(v_{\sigma(\gamma)}\right)_{\gamma \in \Gamma}$, let $n$ be a positive integer, with $n \leq \operatorname{card} \Gamma, \alpha_{1}, \ldots, \alpha_{n}$ distinct elements of $\Gamma$, and for each $i,\left(\beta_{j}^{i}\right)_{j=1}^{m_{t}}$ distinct elements of $\Gamma_{\alpha_{i}}$ (with $m_{i} \leq \operatorname{card} \Gamma_{\alpha_{i}}$ ). Let then scalars $\left(c_{i j}\right)$ be given. We must show that

$$
\left\|\sum_{i=1}^{n} \sum_{j=1}^{m_{i}} c_{i j} v_{\sigma\left(\alpha, \beta_{j}^{i}\right)}\right\|=\left\|\sum_{i=1}^{n} \sum_{j=1}^{m_{i}} c_{i j} u_{\alpha_{i} \beta_{j}^{j}}\right\| .
$$

For each $i$, let

$$
h_{i}=\sum_{j=1}^{m_{i}} c_{i j} u_{\alpha_{i} \beta_{j}^{i}} \text { and } \quad \tilde{h}_{i}=\sum_{j=1}^{m_{i}} c_{i j} v_{\sigma\left(\alpha_{i} \beta_{j}^{\prime}\right)} .
$$


Then

$$
\left\|h_{i}\right\|=\left(\sum_{j=1}^{m_{i}} c_{i j}^{2}\right)^{1 / 2}=\left\|\tilde{h}_{i}\right\| \text { for all } i .
$$

Since $h_{i} \in H_{\alpha_{i}}$, there is an isometry $U$ of $B$ with $U h_{i}=\tilde{h}_{i}$ for all $i$, hence (25) holds.

Finally set $X=\left(\Sigma_{\Lambda} \oplus \tilde{H}_{\beta}\right)_{\underline{v}}$ and suppose $T: B \rightarrow X$ is a surjective isometry. Then it is evident that $T$ induces a bijection $\tilde{T}$ between $\mathscr{H}(B)$ and $\mathscr{H}(X)$, where $\tilde{T}(H)=T H$ for all $H \in \mathscr{H}(B)$. Consequently for each $\alpha \in \Gamma$ there is a unique $\sigma(\alpha)$ in $\Lambda$ with $T H_{\alpha}=\tilde{H}_{\sigma(\alpha)}^{\prime}$ (where the $\tilde{H}_{\beta}^{\prime \prime}$ 's are the canonical images of the $\tilde{H}_{\beta}$ 's in $X$ ). The map $\sigma$ thus defined is a bijection from $\Gamma$ onto $\Lambda$. Since $T$ is an isometry, $\operatorname{dim} \tilde{H}_{\sigma(\alpha)}=\operatorname{dim} \tilde{H}_{\sigma(\alpha)}^{\prime}=$ $\operatorname{dim} H_{\alpha}$ for all $\alpha$. Let us use the notation " " to mean "is isometrically equivalent to." We then have

$$
\left(u_{\alpha}\right)_{\alpha \in \Gamma} \sim\left(T u_{\alpha}\right)_{\alpha \in \Gamma} \sim\left(v_{\sigma(\alpha)}\right)_{\alpha \in \Gamma}
$$

since $T$ is an isometry, $X$ is a functional unconditional sum of $\left\{\tilde{H}_{\sigma(\alpha)}\right.$ : $\alpha \in \Gamma\}$ and $T u_{\alpha}$ is a normalized member of $H_{\sigma(\alpha)}^{\prime}$ for all $\alpha \in \Gamma$. This completes the proof.

Theorem 3.7 easily yields the corresponding classification theorem for complex Banach spaces with a one-unconditional basis. The statement of the result is precisely that of Theorem 3.7, except that "FHS" is replaced by "complex with a one-unconditional basis," the words "with $\operatorname{dim} H_{\alpha} \geq$ 2," are deleted, and the word "real" is inserted before "pure space $U$." Indeed, suppose $B$ is a complex Banach space with a one-unconditional basis and let $X=B_{\mathrm{R}}$. Then $X$ and $B$ have the same Hilbert components (see the discussion at the end of section one). Hence all Hilbert components of $X$ are at least two-dimensional so $X$ is FHS and Theorem 3.7 easily yieldds the complex result. Similar comments apply to our results later on.

Our next result characterizes in particular the Lie algebras of FHS spaces.

THEOREM 3.8. Assume that $X=\mathrm{FH}(B)$ is orthogonally complemented with $Y=\mathcal{O}(\mathrm{FH}(B))$ and let $\mathscr{H}(B)=\left\{H_{\gamma}: \gamma \in \Gamma\right\}$. Suppose for each $\gamma \in \Gamma, T_{\gamma} \in \mathfrak{A}\left(H_{\gamma}\right)$ and $M=\sup _{\gamma}\left\|T_{\gamma}\right\|<\infty$. There is a unique $T \in \mathfrak{A}(B)$ with $T \mid H_{\gamma}=T_{\gamma}$ for all $\gamma \in \Gamma$ and $T y=0$ for all $y \in Y$; moreover $\|T\|=$ $M$. If conversely $T \in \mathfrak{U}(X)$, then $T H_{\alpha} \subset H_{\alpha}$ for all $\alpha \in \Gamma$ and there is $a$ $\tilde{T} \in \mathfrak{A}(B)$ with $\tilde{T} \mid X=T$ and $\tilde{T} \mid Y=0$. 
REMARK. Theorem 3.8 and Corollary 3.3 thus imply that $\mathfrak{A}(B)=$ $\mathfrak{A}(X) \oplus \mathfrak{A}^{\prime}(Y) .\left(\mathfrak{U}^{\prime}(X)\right.$ is defined at the beginning of section two.) They also show that if $B=X$, then $\mathfrak{A}(B)=\left(\Sigma \oplus \mathfrak{X}\left(H_{\alpha}\right)\right)_{l^{\infty}(\Gamma)}$.

Proof of 3.8. Suppose without loss of generality that $M=1$. Since $X$ is a functional unconditional sum of the $H_{\alpha}$ 's, and $X \oplus Y=B$, it follows easily that there is a $T \in \mathscr{L}(B)$ with the desired properties and also $\|T \mid X\|=1$. But then if $x \in X$ and $y \in Y,\|T(x+y)\|=\|T x\| \leq\|x\| \leq$ $\|x+y\|$ since $X \oplus Y$ is an orthogonal decomposition of $B$, hence $\|T\|=1$. To show that $T \in \mathfrak{A}(B)$, let $x$ be a real number. We shall show that $e^{x T}$ is an isometry of $B$. Let $n$ be a positive integer and $\alpha_{1}, \ldots, \alpha_{n}$ distinct members of $\Gamma$. Let $T_{i}=T_{\alpha_{i}}$ and $H_{i}=H_{\alpha_{i}}$ for all $i$. Since $e^{x T_{i}} \in \mathscr{I}\left(H_{i}\right)$, there is a $U_{i} \in \mathscr{I}(B)$ with $U_{i}=e^{x T_{i}}$ on $H_{i}$ and $U_{i}=I$ on $\mathcal{O}\left(H_{i}\right)$ for all $i$, $1 \leq i \leq n$. Then letting $U=U_{1} \cdot \ldots \cdot U_{n}, h_{i} \in H_{i}$ for all $i$ and $y \in Y$, we have that

$$
U\left(h_{1}+\cdots+h_{n}+y\right)=e^{x T}\left(h_{1}+\cdots+h_{n}+y\right),
$$

hence $e^{x T}$ is an isometry since $U$ is an isometry. For the converse, we have that if $T \in \mathfrak{A}(X)$, then $T H_{\alpha} \subset H_{\alpha}$ for all $\alpha$ by Proposition 3.2. Hence by the first part, we obtain the desired $\tilde{T}$. The remark follows from Corollary 3.3 and the fact that $\mathfrak{A}(B)$ is a linear space. Indeed, suppose $T \in \mathfrak{A}(B)$. Then $T \mid X \in \mathfrak{U}(X)$ and $T \mid Y \in \mathfrak{U}{ }^{\prime}(Y)$. But $T \mid X \oplus 0 \in \mathfrak{U}(B)$ by the theorem. Hence since $T=T|X \oplus T| Y, 0 \oplus T \mid Y \in \mathfrak{A}(B)$. Evidently this shows also if $S \in \mathfrak{U}^{\prime}(Y)$, then $0 \oplus S \in \mathfrak{A}(B)$; that is, $\mathfrak{A}(B)$ $=\mathfrak{A}(X) \oplus \mathfrak{A}^{\prime}(Y)$.

We pass next to the proof that pure spaces have a trivial Lie algebra. Together with the preceding result and Theorem 3.6 we then easily obtain a complete description of the Lie algebra of a space with a one-unconditional basis. We first require a simple result characterizing those Lie algebras with rank-two operators.

LEMMA 3.9. Let $X$ and $Y$ be orthogonally complemented one-dimensional subspaces of $B$ with $X$ and $Y$ orthogonal. Let $P$ be the orthogonal projection onto $Z=X+Y$ and $T$ a skew-Hermitian operator on $B$. Then PTP is skew-Hermitian.

REMARK. Thus if PTP is nonzero, $Z$ is a two-dimensional well-embedded Hilbert space, so the lemma characterizes the existence of such a $Z$.

Proof of 3.9. Let $P_{1}$ be the orthogonal projection onto $X$ and $P_{2}$ the orthogonal projection onto $Y$. Then $P_{1} P_{2}=P_{2} P_{1}=0$ and $P=P_{1}+P_{2}$. 
Since $T$ is skew-Hermitian and the $P_{i}$ 's are rank-one, $P_{i} T P_{i}=0$ for $i=1$, 2. Letting $Q=I-P$, then $Q T Q=P_{1} T P_{1}+P_{2} T P_{2}+Q T Q$ is skewHermitian by the Diagonalization Theorem 1.10. But also PTP $+Q T Q$ is skew-Hermitian by Theorem 1.10. Hence PTP is skew-Hermitian since $\mathfrak{A}(B)$ is a linear space.

REMARK. It is worth pointing out that the conclusion of 3.9 fails if the first hypothesis is deleted. Indeed, as we observed in [10], Robbin's theorem (see Theorem 1.4 above) yields that there is a four-dimensional Banach space $\left(\mathbf{R}^{4},\|\cdot\|\right)$ with a one-dimensional Lie algebra spanned by $T=R_{0} \oplus 2 R_{0}$ (with respect to the usual orthonormal basis $\left(e_{1}, \ldots, e_{4}\right)$ for $\mathbf{R}^{4}$; the operators $R_{0}$ and $R(\theta)$ are defined preceding Lemma 2.4 in (10)). Then $e^{\pi T}=R(\pi) \oplus R(2 \pi)=-I \oplus I$ is an isometry. This shows that $X$ is orthogonally complemented by $Y$ in $B$, where $X=\left[e_{1}, e_{2}\right]$ and $Y=\left[e_{3}, e_{4}\right]$. Nevertheless if $P$ is the orthogonal projection on $X, P T P=$ $R_{0} \oplus 0$ is not skew-Hermitian since $B$ has no rank-two skew-Hermitian operators. (Notice also that $X$ and $Y$ are Euclidean; thus $\left[e_{1}\right]$ is orthogonally complemented in $X$ and $X$ is orthogonally complemented in $B$, but $\left[e_{1}\right]$ is not orthogonally complemented in $B$, else $\left[e_{2}\right]$ would also be, and then Lemma 3.10 would apply, yielding PTP skew-Hermitian.)

We are now prepared for the proof that pure spaces have trivial Lie algebras. The proof also gives an alternate proof of Corollary 3.4.

THEOREM 3.10. Let $U$ be a real Banach space with a one-unconditional basis and suppose $U$ is pure; i.e., there are no rank-two operators in $\mathfrak{A}(U)$. Then $\mathfrak{A}(U)=\{0\}$.

Proof. Let $\left(u_{\alpha}\right)_{\alpha \in \Gamma}$ be a normalized one-unconditional basis for $U$, let $\left(u_{\alpha}^{*}\right)_{\alpha \in \Gamma}$ be the functionals in $U^{*}$ biorthogonal to the $u_{\alpha}$ 's and suppose $T \in \mathfrak{A}(U)$ with $T \neq 0$. Choose then $\alpha \in \Gamma$ with $T u_{\alpha} \neq 0$. Now $u_{\alpha}^{*}\left(u_{\alpha}\right)$ $=\left\|u_{\alpha}^{*}\right\|=1$. Since $T$ is skew-Hermitian, $u_{\alpha}^{*} T u_{\alpha}=0$. Since $T u_{\alpha}=$ $\sum_{\beta \in \Gamma} u_{\beta}^{*}\left(T u_{\alpha}\right) u_{\beta}$, there must then be a $\beta \neq \alpha$ with $u_{\beta}^{*} T u_{\alpha} \neq 0$. Also we have that $u_{\beta}^{*} T u_{\beta}=0$. Now $X=\left[u_{\alpha}\right]$ and $Y=\left[u_{\beta}\right]$ satisfy the hypotheses of Lemma 3.9. Thus PTP is skew-Hermitian, where $P$ is the orthogonal projection onto $\left[u_{\alpha}, u_{\beta}\right]$. Of course $P b=u_{\alpha}^{*}(b) u_{\alpha}+u_{\beta}^{*}(b) u_{\beta}$ for all $b \in$ $U$. It follows that

$$
P T P(b)=\left[u_{\beta}^{*}(b) u_{\alpha}^{*}\left(T u_{\beta}\right)\right] u_{\alpha}+\left[\left(u_{\alpha}^{*}(b)\right) u_{\beta}^{*}\left(T u_{\alpha}\right)\right] u_{\beta}
$$

for all $b \in B$. In particular, $\operatorname{PTP}\left(u_{\alpha}\right)=\left(u_{\beta}^{*} T u_{\alpha}\right) u_{\beta} \neq 0$, hence PTP is nonzero. Evidently rank $P T P \leq 2$, so rank $P T P=2$ and $U$ is not pure. 
(We also obtain that in fact $\left[u_{\alpha}, u_{\beta}\right]=$ range $P T P$ is thus a two-dimensional well-embedded Hilbert space, reproving Corollary 3.4.)

Our next result follows immediately from Theorem 3.6, 3.8 and 3.10. It yields the complete description of the Lie algebra of a Banach space with a one-unconditional basis.

COROLlaRY 3.11. Let $B$ have a normalized one-unconditional basis, $X=F H(B)$ and $Y=\mathcal{O}(X)$. Then $\mathfrak{A}^{\prime}(Y)=\{0\}$ and $\mathfrak{A}(B)=\mathfrak{A}(X) \oplus$ $\{0\}$. If moreover $T \in \mathfrak{A}(B)$, then $\|T\|=\|T \mid X\|$. The skew-Hermitian operators on $B$ are thus described by Theorems 3.6 and 3.8.

REMARK. It follows that if $B$ is finite-dimensional, $\mathscr{I}_{0}(B)=\mathscr{I}_{0}(X) \oplus I$ (where as usual, $\mathscr{I}_{0}(Y)$ denotes the component of the identity of $\mathscr{I}(B)$ ).

The following simple example illustrates some of these results. Define a norm $\|\cdot\|$ on $\mathbf{R}^{3}$ by

$$
\left\|x e_{1}+y e_{2}+z e_{3}\right\|=\max \left\{\left(x^{2}+y^{2}\right)^{1 / 2},\left(y^{2}+z^{2}\right)^{1 / 2},\left(x^{2}+z^{2}\right)^{1 / 2}\right\},
$$

where $\left(e_{1}, e_{2}, e_{3}\right)$ is the usual orthonormal basis. Setting $U=\left(\mathbf{R}^{3},\|\cdot\|\right)$, we have that $\left(e_{1}, e_{2}, e_{3}\right)=\underline{u}$ is a one-unconditional normalized basis for $U$; for $i \neq j,\left[e_{i}, e_{j}\right]$ is an orthogonally complemented Euclidean subspace. However this space is not well-embedded. Indeed, it suffices to see that $\left[e_{1}, e_{2}\right]$ is not well-embedded. Since $\mathcal{O}\left[e_{1}, e_{2}\right]=\left[e_{3}\right]$, we simply observe that

$$
\left\|e_{1}+\frac{1}{\sqrt{2}} e_{3}\right\|=\sqrt{\frac{3}{2}}
$$

while

$$
\left\|\frac{e_{1}+e_{2}}{\sqrt{2}}+\frac{e_{3}}{\sqrt{2}}\right\|=1 .
$$

Thus $U$ is pure, so has a trivial Lie algebra. Now define a norm $\|\cdot\|^{\prime}$ on $\mathbf{R}^{4}$ by

$$
\left\|\left(x_{1}, x_{2}, x_{3}, x_{4}\right)\right\|^{\prime}=\left\|\left(x_{1}^{2}+x_{2}^{2}\right)^{1 / 2} e_{1}+x_{3} e_{2}+x_{4} e_{3}\right\|
$$

and set $B=\left(\mathbf{R}^{4},\|\cdot\|^{\prime}\right)$. Evidently $\left(e_{1}, \ldots, e_{4}\right)$ is a one-unconditional basis for $B$. Then setting $X=\left[e_{1}, e_{2}\right], Y=\left[e_{3}, e_{4}\right]$, we have that $X=\operatorname{FH}(B)$ and $Y=\mathcal{O}(X)$. Indeed, it is clear that $X$ is a well-embedded Euclidean subspace of $B$. To see that $X$ is a Hilbert component, by Theorem 3.6 it suffices to show that $\left[e_{1}, e_{2}, e_{i}\right]$ is not well-embedded for $i=3$ or 4 . This 
is easily done as in the discussion of $U$ (however again, $\left[e_{1}, e_{2}, e_{3}\right]$ is Euclidean and of course orthogonally complemented in $B$ ). Also, we easily verify that $\left[e_{3}\right],\left[e_{4}\right]$ are Hilbert components. The pure-space $V$ of Theorem 3.6 is then $\left[e_{1}, e_{3}, e_{4}\right]$ which is of course isometric to $U$. $Y$ is of course Euclidean and orthogonally complemented; thus although $\mathfrak{U}^{\prime}(Y)$ $=0, \mathfrak{A}(Y) \neq 0$.

Detailed structure of FHS spaces and their isometry groups.

We may easily describe the isometries of an FHS space, using Theorem 3.7.

THEOREM 3.12. Let $\left(H_{\alpha}\right)_{\alpha \in \Gamma}$ be Hilbert spaces all of dimension at least two, $\underline{u}=\left(u_{\alpha}\right)_{\alpha \in \Gamma}$ a one-unconditional basis for a pure space $U$, and $B=\left(\Sigma_{\Gamma} \oplus H_{\alpha}\right)_{\underline{u}}$. Let $S(B)$ denote the set of all bijections $\sigma: \Gamma \rightarrow \Gamma$ so that

(a) $\left(u_{\sigma(\alpha)}\right)_{\Gamma}$ is isometrically equivalent to $\left(u_{\alpha}\right)_{\Gamma}$ and

(b) $H_{\sigma(\alpha)}$ is isometric to $H_{\alpha}$ for all $\alpha$.

Let $\sigma$ in $S(B)$. For each $\alpha \in \Gamma$, let $T_{\alpha}: H_{\alpha} \rightarrow H_{\sigma(\alpha)}$ be a surjective linear isometry. There is then a unique $T$ in $\mathscr{I}(B)$ so that for all $x=\left(x_{\alpha}\right)_{\alpha \in \Gamma}$ in $B,(T x)_{\sigma(\alpha)}=T_{\alpha} x_{\alpha}$ for all $\alpha$ in $\Gamma$. Conversely, every $T$ in $\mathscr{I}(B)$ is of this form.

Proof. For ease in notation, let us identify the $H_{\alpha}$ 's with their canonical images in $B$. Let then $x \in B, x=\sum_{\alpha \in \Gamma} x_{\alpha}$ with $x_{\alpha} \in H_{\alpha}$ for all $\alpha$. We shall show first that given $\sigma$ and the $T_{\alpha}$ 's then $\sum_{\alpha \in \Gamma} T_{\alpha}\left(x_{\alpha}\right)$ converges to something in $B$ with the same norm as $x$. For any finite $F \subset \Gamma$,

$$
\left\|\sum_{\alpha \in F}\right\| T_{\alpha}\left(x_{\alpha}\right)\left\|u_{\sigma(\alpha)}\right\|=\left\|\sum_{\alpha \in F}\right\| x_{\alpha}\left\|u_{\alpha}\right\| .
$$

Hence $\sum_{\alpha \in \Gamma}\left\|T_{\alpha}\left(x_{\alpha}\right)\right\| u_{\sigma(\alpha)}$ converges to an element of $U$, so $T x=$ $\sum_{\alpha \in \Gamma} T_{\alpha} x_{\alpha}$ is a well-defined member of $B$ with $\|T x\|=\|x\|$. It is evident that then $T^{-1}$ corresponds to the maps $\sigma^{-1} \in S(B)$ and $T_{\alpha}^{-1}: H_{\beta} \rightarrow H_{\sigma^{-1}(\beta)}$ for all $\beta \in \Gamma$, so $T \in \mathscr{I}(B)$. Conversely, let $T \in \mathscr{I}(B)$. Since $\mathscr{H}(B)=$ $\left\{H_{\alpha}: \alpha \in \Gamma\right\}$, for each $\alpha \in \Gamma$, there is a unique $\sigma(\alpha)$ in $\Gamma$ with $T H_{\alpha}=$ $H_{\sigma(\alpha)}$ by Corollary 3.3. Thus setting $T_{\alpha}=T \mid H_{\alpha}, T_{\alpha}$ is a surjective isometry mapping $H_{\alpha}$ onto $H_{\sigma(\alpha)}$, so $H_{\alpha}$ and $H_{\sigma(\alpha)}$ are isometric; moreover the map $\sigma$ thus defined is a bijection. For each $\alpha \in \Gamma$, let $h_{\alpha}$ be a normalized member of $H_{\alpha}$. Then by Theorem 3.7, we have that $\left(h_{\alpha}\right)_{\alpha \in \Gamma}$ is isometrically equivalent to $\left(u_{\alpha}\right)_{\alpha \in \Gamma}$ and $\left(T h_{\alpha}\right)_{\alpha \in \Gamma}$ is isometrically equivalent to

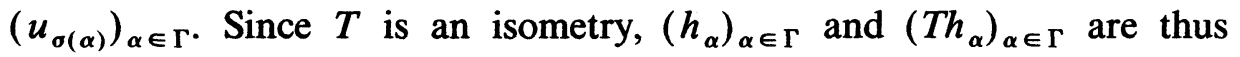
isometrically equivalent, completing the proof. 
Remarks. (1) This result easily extends to the complex case. The statement is exactly the same, except that we drop the requirement that the $H_{\alpha}$ 's are of dimension at least two. This result is essentially known; for finite-dimensional complex $B$, see [11] and for separable complex $B$, see [3].

(2) Let $B$ be a separable $F H S$ space with Hilbert components $\left(H_{\lambda}\right)_{\lambda \in \Gamma}$. It follows from known results and Remark 2 following Proposition 3.2 that then $\mathscr{I}_{0}(B)$ is an arc-wise connected open subgroup of $\mathscr{I}(B)$ and $T \in \mathscr{I}_{0}(B)$ if and only if $T \in \mathscr{I}(B)$ and $T \mid H_{\lambda} \in \mathscr{I}_{0}\left(H_{\lambda}\right)$ for all $\lambda$. (If $H$ is the $n$-dimensional Hilbert space $E_{n}, \mathscr{I}_{0}(H)=S O_{n}$, while by the results of [7], if $H$ is separable infinite-dimensional, $\mathscr{I}_{0}(H)=\mathscr{I}(H)$ is arc-wise connected.)

(3) A one-unconditional normalized basis $\underline{u}=\left(u_{\alpha}\right)_{\alpha \in \Gamma}$ for a real or complex space $U$ is called symmetric if for every bijection $\sigma: \Gamma \rightarrow \Gamma$, there is an isometry $T_{\sigma}$ of $U$ with $T_{\sigma} u_{\alpha}=u_{\sigma(\alpha)}$ for all $\alpha$. It follows immediately from Corollary 3.4 and Theorem 3.6 below that if $\underline{u}$ is symmetric and $U$ is real and non-Euclidean, then $U$ is pure. Theorem 3.12 thus yields a complete description of the isometries of $F H S$ spaces $B=\left(\Sigma_{\Gamma} H_{\alpha}\right)_{\underline{u}}$ for such $\underline{u}$. If the $H_{\alpha}$ 's are all two-dimensional, then $B$ is isometric to $V_{\mathbf{R}}$ where $V$ is a complex non-Euclidean space with a symmetric basis $\left(v_{\alpha}\right)_{\alpha \in \Gamma}$, say. We thus obtain the result that $\mathscr{I}(V)$ and $\mathfrak{A}(V)$ are characterized as follows: Given $T \in \mathscr{I}(V)$, there is a bijection $\sigma: \Gamma \rightarrow \Gamma$ and a $\varphi: \Gamma \rightarrow \mathbf{C}$ with $|\varphi(\gamma)|=1$ for all $\gamma$ so that $T v_{\alpha}=\varphi(\gamma) v_{\sigma(\gamma)}$ for all $\gamma$. Given $S \in \mathfrak{A}(V)$, there is a bounded $\varphi: \Gamma \rightarrow\{a i: a \in \mathbf{R}\}$ with $S v_{\gamma}=\varphi(\gamma) v_{\gamma}$ for all $\gamma$. This result is known (for countable $\Gamma$ ) and due to Tam [12].

Let $X$ be a real or complex Banach space. Say that $X$ has an essentially unique one-unconditional basis if $X$ has a normalized one-unconditional basis $\left(u_{\alpha}\right)_{\alpha \in \Gamma}$ so that if $\left(v_{\alpha}\right)_{\alpha \in \Lambda}$ is another normalized one-unconditional basis, there exists a bijection $\sigma: \Gamma \rightarrow \Lambda$ and for each $\alpha$, a scalar $\lambda_{\alpha}$ with $\left|\lambda_{\alpha}\right|=1$ so that $v_{\sigma(\alpha)}=\lambda_{\alpha} u_{\alpha}$ for all $\alpha$. It follows easily that no FHS space has an essentially unique one-unconditional basis. We observed at the end of section two that if $X$ is a non-Euclidean complex 2-dimensional space, then if $X$ has a one-unconditional basis, this basis is essentially unique. Our next result generalizes this fact. Its proof follows easily from the preceding results and shall be omitted.

COROLlARY 3.13. Let $X$ be a complex Banach space with a normalized one-unconditional basis $\left[u_{\alpha}\right]_{\alpha \in \Gamma}$. The following are equivalent:

(a) The basis is essentially unique. 
(b) The Hilbert components of $X$ are one-dimensional.

(c) $U$ is pure, where $U$ denotes the closed linear span of the $u_{\alpha}$ 's over the real numbers.

Theorem 3.7 classifies FHS spaces isometrically. We now make this classification explicit; we present the results for spaces of arbitrary cardinality. Thus, we present a complete set of isometric invariants for arbitrary FHS spaces.

Let $\Gamma$ be a fixed nonzero cardinal number (which we identify with an initial ordinal, say; that is, $\Gamma$ is the least ordinal number $\eta$ such that card $\Gamma=\operatorname{card} \eta$ (where also $\eta=\{\beta: \beta<\eta\})$ ). Let $\operatorname{PU}(\Gamma)$ (for "Pure Unconditional of cardinality $\Gamma$ ) be the family of all normalized one-unconditional bases $\underline{u}=\left(u_{\gamma}\right)_{\gamma \in \Gamma}$ for some Banach space $U$ such that $U$ is pure. Let $m$ be an infinite cardinal number with $\Gamma \leq \mathfrak{m}$. Let $G(\mathfrak{m})$ denote the set of cardinal numbers $a$ with $1<\mathfrak{a} \leq \mathfrak{m}$. For each $a$ in $G(\mathfrak{m})$, let $H_{\mathrm{a}}=l^{2}(\mathrm{a})$. Thus $H_{\mathrm{a}}$ is a real Hilbert space of dimension $a$. Now given $\underline{u} \in \mathrm{PU}(\Gamma)$ and $\varphi: \Gamma \rightarrow G(\mathrm{~m})$, define

$$
B(\varphi, \underline{u})=\left(\sum_{\alpha \in \Gamma} \oplus H_{\varphi(\alpha)}\right) .
$$

Define an equivalence relation $R$ on $G(\mathfrak{m})^{\Gamma} \times \mathrm{PU}(\Gamma)$ as follows: $(\varphi, \underline{u}) R(\psi, \underline{v})$ if and only if there exists a map $\sigma: \Gamma \rightarrow \Gamma$ which is one-one and onto so that $\left(v_{\sigma(\alpha)}\right)$ is isometrically equivalent to $\left(u_{\alpha}\right)$ and $\psi \circ \sigma=\varphi$. Given $(\varphi, \underline{u})$, let $\langle(\varphi, \underline{u})\rangle$ denote the equivalence class to which $(\varphi, \underline{u})$ belongs. Denote this set of equivalence classes by $\left(G(\mathrm{~m})^{\Gamma} \times \mathrm{PU}(\Gamma)\right) / R$. Our next result shows that the FHS spaces of cardinality at most $m$ are in one-one correspondence (isometrically) with

$$
\bigcup\left\{\left(G(\mathrm{~m})^{\Gamma} \times \mathrm{PU}(\Gamma)\right) / R: 0<\Gamma \leq \mathrm{m}\right\} .
$$

THEOREM 3.14. Let $\mathrm{m}$ be an infinite cardinal number and $B$ in FHS with $\operatorname{dim} B \leq \mathrm{m}$. There exists a unique nonzero cardinal $\Gamma \leq \mathrm{m}$ and $a$ unique $w$ in $\left(G(\mathrm{~m})^{\Gamma} \times \mathrm{PU}(\Gamma)\right) / R$ so that $B$ is isometric to $B(\varphi, \underline{u})$ where $\langle(\varphi, \underline{u})\rangle=w$.

Proof. If $H$ is a Hilbert component of $B$, then $\operatorname{dim} H \leq \operatorname{dim} B \leq \mathfrak{m}$; moreover since the components are orthogonal, it follows that card $\mathscr{H} \leq \mathfrak{m}$, where $\mathscr{H}$ equals the set of Hilbert components of $B$. Thus since every Hilbert space is isometric to $H_{a}$ for some cardinal $a$, we obtain by Theorem 3.7 that there exists a nonzero cardinal $\Gamma \leq \mathfrak{m}$, a $\varphi$ in $G(\mathfrak{m})^{\Gamma}$, 
and a $\underline{u}$ in $\mathrm{PU}(\Gamma)$ with $B$ isometric to $B(\varphi, \underline{u})$ as defined in (26). It follows moreover from Theorem 3.7 that the cardinal $\Gamma$ is uniquely determined. Suppose then $B$ is also isometric to $B(\psi, \underline{v})$ for some $\psi$ in $G(\mathfrak{m})^{\Gamma}$ and $\underline{v}$ in $\mathrm{PU}(\Gamma)$. Thus by Theorem 3.7, there exists a bijection $\sigma$ : $\Gamma \rightarrow \Gamma$ so that $\left(u_{\alpha}\right)_{\alpha \in \Gamma}$ is isometrically equivalent to $\left(v_{\sigma(\alpha)}\right)_{\alpha \in \Gamma}$ and $\operatorname{dim} H_{\psi(\sigma(\alpha))}=\operatorname{dim} H_{\varphi(\alpha)}$ for all $\alpha \in \Gamma$. But for cardinals $\mathfrak{a}$ and $\mathfrak{b}$, $\operatorname{dim} H_{\mathrm{a}}=\operatorname{dim} H_{\mathfrak{b}}$ implies $\mathfrak{a}=\mathfrak{b}$. Hence $\psi \circ \sigma=\varphi$ and $(\varphi, \underline{u}) R(\psi, \underline{v})$, showing the uniqueness of $w$. (Also, if conversely $(\varphi, \underline{u}) R(\psi, \underline{v})$, it is easily proved by the argument for Theorem 3.12 that $B(\varphi, \underline{u})$ is isometric to $B(\psi, \underline{v})$; thus the isometric-type is "well-defined" by $w$.

Remarks. (1) Call $B$ a Generalized Functional Hilbertian Sum if $B$ is a functional unconditional sum of Hilbert spaces, all but at most one of dimension at least two. It is evident that $B$ is GFHS if either $B$ is FHS or $\operatorname{dim} B / F H(B)=1$ and $F H(B)$ is well-embedded in $B$. The theory that we have presented above easily generalizes to GFHS spaces. For example they have isometrically unique unconditional bases; the appropriate analogues of Theorems 3.7, 3.12, and 3.14 hold. The essential new point is that if $B$ is GFHS but not FHS, $B$ has one one-dimensional component $[x]$ say; then if $T \in \mathscr{I}(B), T x= \pm x$. We mention this class of spaces mainly because they include the rotation spaces discussed in section two.

(2) The complex version of Theorem 3.14 follows easily from 3.14. The formulation is almost the same, except that one replaces $G(\mathfrak{m})$ by $G(\mathfrak{m}) \cup\{1\}$, denoted by $\underline{G}(\mathfrak{m})$, say. Then simply let $H_{\mathrm{a}}$ denote the complex Hilbert space $l^{2}(\mathfrak{a})$. For $\varphi$ in $\underline{G}(\mathfrak{m})^{\Gamma}$, the space $B(\varphi, \underline{u})$ is defined exactly as in (26). Now one obtains the classification of complex Banach spaces $B$ with a one-unconditional basis by replacing $G(\mathfrak{m})$ by $\underline{G}(\mathrm{~m})$ in the statement of Theorem 3.14.

(3) It is possible to deduce some of our results from their complex analogues. We indicate here the connection between the real and complex versions. Suppose $\underline{u}=\left(u_{\alpha}\right)_{\alpha \in \Gamma}$ is a normalized one-unconditional basis for a real Banach space $U$. Let $U(\mathbf{C}, \underline{u})$ denote the complex Banach space consisting of all $x \in \mathbf{C}^{\Gamma}$ with $\sum_{\alpha \in \Gamma}\left|x_{\alpha}\right| u_{\alpha} \in U$, under the norm $\|x\|=$ $\left\|\sum_{\alpha \in \Gamma}\left|x_{\alpha}\right| u_{\alpha}\right\|$. Let $\left(u_{\alpha}^{\prime}\right)_{\beta}=0$ if $\beta \neq \alpha,\left(u_{\alpha}^{\prime}\right)_{\alpha}=1$. Then of course $\left(u_{\alpha}^{\prime}\right)_{\Gamma}$ is a one-unconditional basis for $U(\mathbf{C}, \underline{u})$. We may assume without loss of generality that $u_{\alpha}=u_{\alpha}^{\prime}$ for all $\alpha$. Given $T \in \mathscr{L}(U)$, define $\tilde{T} \in$ $\mathscr{L}(U(\mathbf{C}, \underline{u}))$ as follows: Given $x=\left(a_{\alpha}+i b_{\alpha}\right)_{\alpha \in \Gamma}$ in $U(\mathbf{C}, \underline{u})$, let $\tilde{T} x=$ $T\left(\sum_{\alpha \in \Gamma} a_{\alpha} u_{\alpha}\right)+i T\left(\sum_{\alpha \in \Gamma} b_{\alpha} u_{\alpha}\right)$. In fact, we have that $\|\tilde{T}\| \leq 2\|T\|$. It is evident that if $\tilde{T} \in \mathscr{I}(u(\mathbf{C}, \underline{u})), T \in \mathscr{I}(U)$. The converse is of course false in general. Nevertheless it can be shown that for $T \in \mathscr{L}(U), T \in \mathfrak{A}(U)$ if 
and only if $\tilde{T} \in \mathfrak{A}(U(\mathbf{C}, \underline{u}))$. (This can be proved directly; however it does follow from our results above.) Assuming the complex form of the decomposition given in Theorem 3.6, the "real parts" of the Hilbert components of $U(\mathbf{C}, \underline{u})$ may then be used to obtain Theorem 3.6 itself. Suppose $\underline{v}=\left(v_{\alpha}\right)_{\alpha \in \Gamma}$ is a normalized one-unconditional basis for a real space $V$. If $U(\mathbf{C}, \underline{u})$ and $V(\mathbf{C}, \underline{v})$ are isometric, it follows by the complex version of Theorem 3.7 that $\underline{u}$ and $\underline{v}$ are isometrically equivalent. It is false, in general, that $U$ and $V$ isometric implies $U(\mathbf{C}, \underline{u})$ and $V(\mathbf{C}, \underline{v})$ isometric since $\underline{u}$ and $\underline{v}$ need not be isometrically equivalent. However it follows by our results that this is true if $U$ and $V$ are FHS spaces. In this case, we thus obtain that $U$ is isometric to $V$ if and only if $U(\mathbf{C}, \underline{u})$ is isometric to $V(\mathbf{C}, \underline{v})$, and hence Theorems 3.7 and 3.12 may be deduced from their complex versions.

\section{Complementation results.}

We conclude this section with some results dealing with one-complemented subspaces of spaces with a one-unconditional basis. Our first result in this direction gives a certain characterization of those one-complemented subspaces of FHS spaces which are again FHS. (Recall that for $X \subset B, X$ is one-complemented if there is a norm-one projection mapping $B$ onto $X$.) To motivate it, suppose $B$ is FHS. $B$ has the following property:

$$
\text { For all } b \in B \text {, there is } a T \in \mathfrak{U}(B) \text { with } T^{2} b=-b .
$$

(We note in passing that if $B$ is an arbitrary Banach space satisfying (27), then $T$ can be chosen independently of $y$ in (27) if and only if $B$ is isometric to a complex Banach space, by Theorem 2.1 of [10]. For $B$ in FHS, this evidently occurs if and only if the Hilbert components of $B$ are either infinite or of even finite dimension.) To see that (27) holds, let $\left(H_{\lambda}\right)_{\lambda \in \Lambda}$ be the Hilbert components of $B$. Let $y \in B$, choose $y_{\lambda} \in H_{\lambda}$ with $y=\sum_{\lambda \in \Lambda} y_{\lambda}$. For each $\lambda \in \Lambda$, choose $T_{\lambda}$ a rank-2 member of $\mathfrak{U}\left(H_{\lambda}\right)$ with $\left\|T_{\lambda}\right\|=1$ and $T_{\lambda}^{2} y_{\lambda}=-y_{\lambda}$. Then evidently $T=\sum_{\lambda \in \Lambda} \oplus T_{\lambda}$ has the desired properties. ( $T$ is well defined by Theorem 3.8.)

The above discussion and our next result show in particular that $B$ is FHS if and only if $B$ has a one-unconditional basis and the property given in (27). We prove a stronger result later.

THEOREM 3.15. Let $B$ have a one-unconditional basis and $X$ be $a$ one-complemented subspace of $B$. Suppose for all $x \in X$, there is $a T \in \mathfrak{U}(B)$ 
with $T x \in X$ and $T^{2} x=-x$. Then $X$ is FHS (so of course has a one-unconditional basis).

Proof. We shall show that $X$ is the closed linear span of the ranges of the rank-two members of its Lie algebra; hence $X$ is FHS by Theorem 3.1. We first show that we may assume $B$ is FHS. Indeed, by Corollary 3.11 we may write $B=B_{1} \oplus B_{2}$ where $B_{1}=\mathrm{FH}(B), B_{2}=\mathcal{O}\left(B_{1}\right)$ and $\mathfrak{A}(B)$ $=\mathfrak{A}\left(B_{1}\right) \oplus\{0\}$. Our hypotheses then imply $X \subset B_{1}$. For let $x \in X$, $x=b_{1}+b_{2}$ with $b_{i} \in B_{i}$ for $i=1,2$. Choose $T \in \mathfrak{A}(B)$ with $T^{2} x=-x$. But $T x=T b_{1}$ since $T \mid B_{2}=0$. Since $B_{1}$ is invariant under $T$, we have that $T x \in B_{1}$, hence so is $-x=T^{2} x$.

Now assume $B$ is FHS. Let $\left(H_{\lambda}\right)_{\lambda \in \Lambda}$ be the Hilbert components of $B$. Let $P: B \rightarrow X$ be a norm-one projection and let $x \in X$. Finally, choose $x_{\lambda} \in H_{\lambda}$ for all $\lambda$ with $x=\sum_{\lambda \in \Lambda} x_{\lambda}$. Now choose $T \in \mathfrak{A}(B)$ as in the hypothesis of 3.15. For each $\lambda, T H_{\lambda} \subset H_{\lambda}$ by Proposition 3.2 and hence $\left[x_{\lambda}, T x_{\lambda}\right]$ is orthogonally complemented in $B$ since it is a subspace of $H_{\lambda}$; let $Q_{\lambda}$ be the corresponding orthogonal projection. (Of course $Q_{\lambda} \mid H_{\lambda}$ is in fact the Hilbert-space orthogonal projection and $Q_{\lambda}(b)=0$ for all $b \in H_{\beta}$ for all $\beta \neq \lambda$.)

We have that

$$
x=-T^{2} x=\sum_{\lambda \in \Lambda}-T^{2} x_{\lambda} .
$$

Since $H_{\lambda}$ is invariant under $T,-T^{2} x_{\lambda} \in H_{\lambda}$ for all $\lambda$. It follows from (28) by uniqueness of decompositions with respect to $\left(H_{\lambda}\right)_{\lambda \in \Lambda}$ that

$$
-T^{2} x_{\lambda}=x_{\lambda} \text { for all } \lambda \text {. }
$$

Now let $y=-T x=\Sigma_{\lambda \in \Lambda}-T x_{\lambda}$. Fixing $\lambda \in \Lambda$, we have demonstrated that $\left[x_{\lambda}, T x_{\lambda}\right]$ is invariant under $T$; since this space is the range of $Q_{\lambda}$, we have that $-T x_{\lambda}=Q_{\lambda} y$ and hence $Q_{\lambda} T Q_{\lambda} y=Q_{\lambda}\left(-T^{2} x_{\lambda}\right)=x_{\lambda}$ by (29). Thus $x=\sum_{\lambda \in \Lambda} Q_{\lambda} T Q_{\lambda} y$, so

$$
P x=x=\sum_{\lambda \in \Lambda} P Q_{\lambda} T Q_{\lambda} y .
$$

Again fixing $\lambda \in \Lambda, Q_{\lambda} T Q_{\lambda} \in \mathfrak{U}(B)$ by Theorem 3.8 (since $Q_{\lambda} T Q_{\lambda} \mid H_{\lambda} \in$ $\left.\mathfrak{A}\left(H_{\lambda}\right)\right)$. Thus by Proposition 1.2, the compression of $Q_{\lambda} T Q_{\lambda}$ to $X$ via $P$, namely $P Q_{\lambda} T Q_{\lambda} \mid X$, belongs to $\mathfrak{A}(X)$. Since $Q_{\lambda}$ is rank 2, rank $P Q_{\lambda} T Q_{\lambda} \mid X=2$ or $P Q_{\lambda} T Q_{\lambda} \mid X=0$. (30) thus shows that $X$ is in the closed linear span of the ranges of the rank-two members of $\mathfrak{A}(X)$, completing the proof. 
Theorem 3.1 and the preceding result yield the following known result for complex spaces (cf. [9] for a relatively short complex-scalars proof):

Corollary 3.16. (Kalton and Wood [6].) Let B be a complex Banach space. Assume $B=[T B: \operatorname{rank} T=1$ and $T \in \mathfrak{A}(B)]$. Then $B$ has a one-unconditional basis (and conversely). If $X$ is a one-complemented subspace of $B, X$ has a one-unconditional basis.

Proof. Let $Y=B_{\mathbf{R}}$; i.e., $B$ regarded as a real Banach space. Now assuming the first hypothesis, $Y$ is FHS. Indeed, if $T \in \mathfrak{A}(B)$ and $T$ is rank-one as a complex linear operator, then $T \in \mathfrak{A}(Y)$ and $T$ is rank-two over the reals. Now if $H$ is a Hilbert component of $Y$, then as pointed out at the end of section one, $H$ is a complex linear subspace and in fact a Hilbert component of $B$; in particular, $H$ is orthogonally complemented. Thus if $\left\{H_{\lambda}\right\}_{\lambda \in \Lambda}$ are the Hilbert components of $Y$, then the $H_{\lambda}$ 's are orthogonal in $B$. If for each $\lambda,\left(e_{\lambda \beta}\right)_{\beta \in \Gamma_{\lambda}}$ is a one-unconditional normalized basis for the complex space $H_{\lambda}$ (i.e., a complete orthonormal system for $H_{\lambda}$ ), then we obtain that $\left\{e_{\lambda \beta}: \beta \in \Gamma_{\lambda}, \lambda \in H_{\lambda}\right\}$ is a one-unconditional basis for $B$.

Now suppose $X$ is one-complemented in $B$. Then $X_{\mathbf{R}}$ trivially satisfies the assumptions of Theorem 3.15. Thus $X_{\mathbf{R}}$ is FHS. Our argument above showed that $B$ has a complex one-unconditional basis just assuming $B_{\mathbf{R}}$ is FHS, so we obtain that $X$ has a one-unconditional basis.

Our next result shows in particular that if $B$ is a complex Banach space so that $B_{\mathbf{R}}$ has a one-unconditional basis, then $B$ has a one-unconditional basis. (This could also be deduced from Theorem 3.1.) It provides yet another characterization of FHS spaces.

Proposition 3.17. Let $B$ be a real Banach space satisfying (27).

(a) Every Hilbert component of $B$ is at least two-dimensional.

(b) Suppose $B$ is the closed linear span of its one-dimensional orthogonally complemented subspaces. Then $B$ is FHS.

Proof. Let $b \in B$ with $\|b\|=1$ and choose $T \in \mathfrak{U}(B)$ with $T^{2} b=-b$. Let $X=[b, T b]$. Then $X$ is invariant under $T$ and $(T \mid X)^{2}=-I \mid X$. Now suppose [b] is orthogonally complemented in $B$ and let $Z$ be a one-dimensional subspace of $X$. We may choose $\alpha$ and $\beta$ with $\alpha^{2}+\beta^{2}=1$ and $Z=[x]$ where $x=\alpha b+\beta T b=(\alpha I+\beta T) b$. Now choose $\theta$ with $\alpha=$ $\cos \theta$ and $\beta=\sin \theta$. Then $e^{\theta T}|X=(\alpha I+\beta T)| X$, hence $e^{\theta T} b=x$. Since $e^{\theta T} \in \mathscr{I}(B),[x]$ is orthogonally complemented in $B$. Thus every one-dimensional subspace of $X$ is orthogonally complemented in $B$, so $X$ is a 
well-embedded Euclidean subspace of $B$ by Theorem 2.2. This proves (a). If we assume (b) holds, then we obtain $B$ is the closed linear span of its two-dimensional well-embedded Euclidean subspaces. Thus, $B=[T B$ : $T \in \mathfrak{A}(B), \operatorname{rank} T=2$ ], so $B$ is FHS by Theorem 3.1.

REmark. Suppose $B$ satisfies the hypothesis (b) of Proposition 3.17. Does $B$ have an unconditional basis? We are able to exhibit examples of finite dimensional $B$ satisfying (b) with no one-unconditional basis (for $\operatorname{dim} B>3$ ).

We end this section with a result concerning orthogonally complemented spaces.

THEOREM 3.18. Let $X$ be an orthogonally complemented subspace of $B$.

(a) If $B$ is real and an FHS space, $X$ has a one-unconditional basis.

(b) If $B$ is complex, $X$ has a one-unconditional basis which can be extended to a one-unconditional basis for $B$.

\section{REMARKS.}

(1) It is trivial that conversely any space with one-unconditional basis is orthogonally complemented in some FHS space. Also, the converse of (b) is trivial.

(2) Our proof for (a) easily generalizes to GFHS spaces (as defined after Theorem 3.14).

(3) The conclusion of (b) does not hold for general FHS spaces. Indeed, suppose $B=\left(\sum_{\Gamma} \oplus H_{\alpha}\right)_{\underline{u}}$ and $S(B)$ are as in Theorem 3.12. We shall show below that the conclusion of (b) holds for all orthogonally complemented subspaces of $B$ precisely when $\sigma^{2}=I_{\Gamma}$ and $\sigma \in S(B)$ implies $\sigma=I_{\Gamma}$. It can also happen that $B$ is complex with a one-unconditional basis and $X$ is a complex subspace which is real-orthogonally complemented in $B$, yet $X_{\mathbf{R}}$ has no one-unconditional basis which can be extended to a one-unconditional basis for $B_{\mathbf{R}}$. (Of course then $X$ is not complex-orthogonally complemented in $B$.)

The proof of 3.18 follows in a straightforward way from the description of isometries of FHS spaces given by Theorem 3.12. In fact, we can give the explicit description of an arbitrary orthogonal projection on such a space.

Proof of 3.18. Assume $B$ is real and let $\left(H_{\alpha}\right)_{\alpha \in \Gamma}$ be the Hilbert components of $B$; for each $\alpha \in \Gamma$, let $u_{\alpha}$ be a normalized member of $H_{\alpha}$

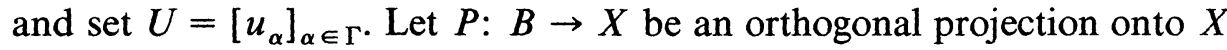
and $T=2 P-I$. Then $T \in \mathscr{I}(B)$ and $X=\{x \in B: T x=x\}$. We now 
use Theorem 3.12 to obtain the structure of $T$. By 3.12, we may choose a bijection $\sigma: \Gamma \rightarrow \Gamma$ with $\left(u_{\sigma(\alpha)}\right)_{\alpha \in \Gamma}$ isometrically equivalent to $\left(u_{\alpha}\right)_{\alpha \in \Gamma}$ with $T H_{\alpha}=H_{\sigma(\alpha)}$ for all $\alpha \in \Gamma$. Since $T^{2}=I$, we thus obtain

$$
\sigma^{2}=I_{\Gamma}
$$

Now let $\Gamma_{1}=\{\alpha \in \Gamma: \sigma(\alpha)=\alpha\}$ and $\Gamma_{2}=\Gamma \sim \Gamma_{1}$. It follows from (31) that we may choose a subset $\Lambda$ of $\Gamma_{2}$ so that

$$
\Gamma_{2}=\Lambda \cup \sigma(\Lambda) \text { and } \Lambda \cap \sigma(\Lambda)=\varnothing .
$$

Set $\Gamma^{\prime}=\Gamma_{1} \cup \Lambda$. For each $\alpha \in \Gamma_{1}$, let $X_{\alpha}=P H_{\alpha}$. Note that $H_{\alpha}$ is invariant under $P$ and thus $X_{\alpha}$ is Euclidean. Moreover $X_{\alpha}=X \cap H_{\alpha}$. For each $\lambda \in \Lambda$, let $X_{\lambda}=\left\{h+T h: h \in H_{\lambda}\right\}$. We shall prove momentarily that $X_{\lambda}$ is also Euclidean; then we shall establish that $\left(X_{\alpha}\right)_{\alpha \in \Gamma^{\prime}}$ is a functional unconditional decomposition of $X$, showing that $X$ has a one-unconditional basis (and also incidentally that $X$ is FHS provided the nonzero $X_{\alpha}$ 's all have dimension at least equal to two).

For each $\lambda \in \Lambda$, define $v_{\lambda}$ and $\alpha(\lambda)$ by

$$
\alpha(\lambda)=\left\|u_{\lambda}+u_{\sigma(\lambda)}\right\| \text { and } v_{\lambda}=(\alpha(\lambda))^{-1}\left(u_{\lambda}+u_{\sigma(\lambda)}\right) .
$$

For $h \in H_{\lambda}$, we have that

$$
\|h+T h\|=\|\| h\left\|u_{\lambda}+\right\| T h\left\|u_{\sigma(\lambda)}\right\|=\|h\|\left\|u_{\lambda}+u_{\sigma(\lambda)}\right\|=\alpha(\lambda)\|h\|,
$$

using that $T$ is an isometry and the fact that the $H_{\alpha}$ 's are a functional unconditional decomposition of $B$. (33) shows that $X_{\lambda}$ is Euclidean. Now let $v_{\alpha}=u_{\alpha}$ for all $\alpha \in \Gamma_{1} ;(32)$ yields that $\left(v_{\alpha}\right)_{\alpha \in \Gamma^{\prime}}$ is a one-unconditional basis for its closed linear span. (33) yields that

$$
\|h\| u_{\lambda}+\|T h\| u_{\sigma(\lambda)}=\|x\| v_{\lambda} \quad \text { if } x=h+T h \text { with } h \in H_{\lambda} .
$$

We next show that $\left(X_{\alpha}\right)_{\alpha \in \Gamma^{\prime}}$ is a functional unconditional decomposition of its closed linear span. Let $x_{\alpha} \in X_{\alpha}$ for all $\alpha \in \Gamma^{\prime}$ with only finitely many $\alpha$ 's nonzero. For each $\lambda \in \Lambda$, choose $h_{\lambda} \in H_{\lambda}$ with $x_{\lambda}=h_{\lambda}+T h_{\lambda}$. Since $\left(H_{\alpha}\right)_{\alpha \in \Gamma}$ is a functional unconditional decomposition of $B$, we obtain that

$$
\begin{aligned}
\left\|\sum_{\alpha \in \Gamma^{\prime}} x_{\alpha}\right\| & =\left\|\sum_{\alpha \in \Gamma_{1}}\right\| x_{\alpha}\left\|u_{\alpha}+\sum_{\lambda \in \Lambda}\right\| h_{\lambda}\left\|u_{\lambda}+\right\| T h_{\lambda}\left\|u_{\sigma(\lambda)}\right\| \\
& =\left\|\sum_{\alpha \in \Gamma^{\prime}}\right\| x_{\alpha}\left\|v_{\alpha}\right\|
\end{aligned}
$$

by (34). 
Since $T^{2}=I$, we obtain by (31) that $X_{\alpha} \subset X$ for all $\alpha \in \Gamma^{\prime}$. To complete the proof that $X$ has a one-unconditional basis, we need only establish that $X=\left[X_{\alpha}\right]$. Now we have that for all $\lambda \in \Lambda$ and $\alpha \in \Gamma_{1}$,

$$
H_{\lambda}+H_{\sigma(\lambda)} \text { is invariant under } T \text { and also } H_{\alpha} \text { is invariant under } T \text {. }
$$

Moreover, we have that

$$
X_{\lambda}=\left\{y \in H_{\lambda}+H_{\sigma(\lambda)}: T y=y\right\} \quad \text { for all } \lambda \in \Lambda .
$$

Indeed, if $\lambda \in \Lambda$ and if $y=y_{1}+y_{2}$ with $y_{1}$ in $H_{\lambda}$ and $y_{2}$ in $H_{\sigma(\lambda)}$, then since $T H_{\sigma(\lambda)}=H_{\lambda}$, if $T y=y$, since $T y=T y_{2}+T y_{1}, T y_{2}=y_{1}$ and $y_{2}=$ $T y_{1}$ because $H_{\lambda}+H_{\sigma(\lambda)}$ is a direct sum.

Now let $x \in X$. Choose for all $\alpha \in \Gamma_{1}$ and $\lambda \in \Lambda$, elements $y_{\alpha} \in H_{\alpha}$, $y_{\lambda} \in H_{\lambda}$ and $y_{\sigma(\lambda)} \in H_{\sigma(\lambda)}$ so that

$$
x=\sum_{\alpha \in \Gamma_{1}} y_{\alpha}+\sum_{\lambda \in \Lambda} y_{\lambda}+y_{\sigma(\lambda)},
$$

the indexed families converging unconditionally to $x$. It follows, since (37) holds and $T x=x$, that

$$
x=\sum_{\alpha \in \Gamma_{1}} T y_{\alpha}+\sum_{\lambda \in \Lambda} T\left(y_{\lambda}+y_{\sigma(\lambda)}\right) .
$$

Since $\left(H_{\alpha}\right)_{\alpha \in \Gamma}$ is an unconditional decomposition of $X$, we obtain by (35), (37) and (38) that $y_{\alpha}=T y_{\alpha}$ and $T\left(y_{\lambda}+y_{\sigma(\lambda)}\right)=y_{\lambda}+y_{\sigma(\lambda)}$ for all $\alpha \in \Gamma_{1}$ and $\lambda \in \Lambda$. Finally, we obtain by (36) and the definition of the $X_{\alpha}$ 's for $\alpha \in \Gamma_{1}$ that $y_{\alpha} \in X_{\alpha}$ and $y_{\lambda}+y_{\sigma(\lambda)} \in X_{\lambda}$ for $\alpha \in \Gamma_{1}$ and $\lambda \in \Lambda$, completing the proof of part (a).

The above argument is of course reversible: that is, suppose $\sigma \in S(B)$ with $\sigma^{2}=I_{\Gamma}$. Choose $\Gamma_{1}$ and $\Lambda$ as above. For each $\alpha \in \Gamma_{1}$, let $X_{\alpha}$ be a subspace of $H_{\alpha}$ and for each $\lambda \in \Lambda$, let $T_{\lambda}: H_{\lambda} \rightarrow H_{\sigma(\lambda)}$ be a surjective isometry; then let $X_{\lambda}=\left\{h+T_{\lambda} h: h \in H_{\lambda}\right\}$. We obtain that $X=$ $\left[X_{\alpha}\right]_{\alpha \in \Gamma^{\prime}}$ is orthogonally complemented, where $\Gamma^{\prime}=\Gamma_{1} \cup \Lambda$. Indeed, since the $H_{\alpha}$ 's are Hilbert spaces, for each $\alpha \in \Gamma_{1}$ we may choose $T_{\alpha} \in \mathscr{I}\left(H_{\alpha}\right)$ with $T_{\alpha}^{2}=I$ and $X_{\alpha}=\left\{x \in H_{\alpha}: T_{\alpha} x=x\right\}$. For each $\lambda \in \Lambda$, let $T_{\sigma(\lambda)}=$ $T_{\lambda}^{-1}$. We then obtain a unique $T \in \mathscr{I}(B)$ with $T \mid H_{\alpha}=T_{\alpha}$ for all $\alpha \in \Gamma$; it is easily seen that $T^{2}=I$ and $X=\{b \in B: T b=b\}$.

We next observe that $X$ has a one-unconditional basis extendable to a one-unconditional basis of $B$ if and only if $\sigma=I_{\Gamma}, \sigma$ as defined above. Indeed, let $\Gamma_{1}$ and $\Lambda$ be as defined above. Suppose $X$ has an extendable one-unconditional basis. Then by Lemma 3.5, every element of the basis must belong to a Hilbert component of $B$. We thus obtain that

$$
X=\left[X \cap H_{\alpha}: \alpha \in \Gamma\right] \text {. }
$$


But it follows easily that $X \cap H_{\lambda}=X \cap H_{\sigma(\lambda)}=\{0\}$ for all $\lambda \in \Lambda$, and $X \cap H_{\alpha}=X_{\alpha}$ for $\alpha \in \Gamma_{1}$. Thus by (39), $X=\left[X_{\alpha}\right]_{\alpha \in \Gamma_{1}}$, hence $\Lambda=\varnothing$, $\Gamma_{1}=\Gamma$ and $\sigma=I_{\Gamma}$. The converse assertion is straightforward. Thus assume $\sigma=I_{\Gamma}$ and let the $X_{\alpha}$ 's be as above. For each $\alpha \in \Gamma$, let $\left(x_{\beta}\right)_{\beta \in \Gamma_{\alpha}^{1}}$ be a normalized one-unconditional basis for $X_{\alpha}$ and $\left(x_{\beta}\right)_{\beta \in \Gamma_{\alpha}^{2}}$ be a normalized one-unconditional basis for the orthogonal complement of $X_{\alpha}$ in $H_{\alpha}$. (If $X_{\alpha}=0$, let $\Gamma_{\alpha}^{1}=\varnothing$; if $X_{\alpha}=H_{\alpha}$, let $\Gamma_{\alpha}^{2}=\varnothing$.) Assume $\Gamma_{\alpha}^{i} \cap \Gamma_{\beta}^{j}$ $=\varnothing$ if $i \neq j$ or $\alpha \neq \beta$. Then simply let $\Gamma_{1}=\bigcup_{\alpha \in \Gamma} \Gamma_{\alpha}^{1}$ and $\Gamma_{2}=\bigcup_{\alpha \in \Gamma} \Gamma_{\alpha}^{2}$; $\left\{x_{\alpha}\right\}_{\alpha \in \Gamma_{1}}$ is then a one-unconditional basis for $X$ and $\left\{x_{\alpha}\right\}_{\alpha \in \Gamma_{2}}$ is a one-unconditional basis for $Y$ while $\left\{x_{\alpha}\right\}_{\alpha \in \Gamma_{1} \cup \Gamma_{2}}$ is a one-unconditional basis for $B$.

If we assume $B$ is a complex space and still define our maps as above, we then obtain the conclusion of (b) once we establish that $\sigma=I_{\Gamma}$. (For instance, the argument of the preceding paragraph yields a (complex) extendable unconditional basis for $X$.) Let then $P: B \rightarrow X$ be an orthogonal projection and $T=2 P-I$. We shall show that $T$ is in the component of the identity of $\mathscr{I}(B)$, i.e., in $\mathscr{I}_{0}(B)$. It then follows from Remark 2 following Proposition 3.2 that $T H_{\alpha} \subset H_{\alpha}$ for all $\alpha$, where $\left(H_{\alpha}\right)_{\alpha \in \Gamma}$ are the Hilbert components of $B$, so $\sigma=I_{\Gamma}$ and hence (b) is established. For each complex $\alpha$ with $|\alpha|=1$, define $U_{\alpha}$ by

$$
U_{\alpha}=P+\alpha(I-P)=(1-\alpha) P+\alpha I .
$$

By the definition of orthogonal complementation, $U_{\alpha} \in \mathscr{I}(B)$ for all $\alpha$ and evidently (40) yields that $U_{1}=I$ and $U_{-1}=T$. Now let $f(\theta)=$ $U_{\cos \theta+\imath \sin \theta}$ for $0 \leq \theta \leq \pi$. Then $f$ is a continuous path in $\mathscr{I}(B)$ joining $I$ to $T$, so $T \in \mathscr{I}_{0}(B)$ and the proof is complete.

REMARK. It is possible to give an "elementary" proof of Theorem 3.18(b); that is, a proof which does not pass through the structure theorem characterizing the group of isometries of complex Banach spaces with a one-unconditional basis, and which deals purely with complex spaces. The proof can be given in the same spirit as the streamlined-argument given in [9].

\section{REFERENCES}

[1] E. Berkson, Hermitian projections and orthogonality in Banach spaces, Proc. London Math. Soc., (1) 24 (1972), 101-118.

[2] F. F. Bonsall, and J. Duncan, Numerical ranges of operators on normed spaces and of elements of normed algebras, Vols. I (1971) and II (1974), Cambridge University Press. 
[3] R. J. Fleming, and J. E. Jamison, Hermitian and abelian operators on certain Banach spaces, Pacific J. Math., 52 (1974), 67-85.

[4] , Isometries on certain Banach spaces, J. London Math. Soc., (2) 9 (1974), 121-127.

[5] S. Helgason, Differential Geometry, Lie Groups and Symmetric Spaces, Academic Press, New York, 1978.

[6] N. J. Kalton, and G. V. Wood, Orthonormal systems in Banach spaces and their applications, Math. Proc. Camb. Phil. Soc., 79 (1976), 493-510.

[7] C. Putnam, and A. Wintner, The orthogonal group in Hilbert space, Amer. J. Math., 74 (1952), 52-78.

[8] J. W. Robbin, Lie Algebras of Infinitesimal Norm Isometries, Linear Algebra Appl., 10 (1975), 95-102.

[9] H. P. Rosenthal, On one-complemented subspaces of complex Banach spaces with a one-unconditional basis, according to Kalton and Wood, Israel Seminar on Geometrical Aspects of Functional Analysis, Meeting No. IX, University of Tel Aviv, 1983-84.

[10] The Lie Algebra of a Banach Space, Proceedings of the Missouri Conference on the Geometry of Banach spaces, Springer Verlag Lecture Note Series, to appear.

[11] H. Schneider, and R. E. L. Turner, Matrices Hermitian for an absolute norm, Linear and Multilinear Algebra, 1 (1973), 9-31.

[12] K. W. Tam, Isometries of certain function spaces, Pacific J. Math., 31 (1969), 233-246.

[13] A. E. Tong, Diagonal submatrices of matrix maps, Pacific J. Math., 32 (1970), 551-559.

[14] I. Vidav, The group of isometries and the structure of finite-dimensional Banach spaces, Linear Algebra Appl., 14 (1976), 227-236.

[15] F. W. Warner, Foundations of Differential Manifolds and Lie Groups, Scott, Foresman; Glenview, Illinois, 1971.

Received March 4, 1985. This work was supported in part by NSF-MCS-8303534.

UNIVERSITY OF TEXAS

Austin, TX 78712 



\section{PACIFIC JOURNAL OF MATHEMATICS EDITORS}

\author{
V. S. VARADARAJAN \\ (Managing Editor) \\ University of California \\ Los Angeles, CA 90024 \\ Herbert Clemens \\ University of Utah \\ Salt Lake City, UT 84112 \\ R. FINN \\ Stanford University \\ Stanford, CA 94305
}

\author{
HERMANN FLASCHKA \\ University of Arizona \\ Tucson, AZ 85721 \\ RAMESH A. GANGOLLI \\ University of Washington \\ Seattle, WA 98195 \\ VAUGHAN F. R. JONES \\ University of California \\ Berkeley, CA 94720 \\ ROBION KIRBY \\ University of California \\ Berkeley, CA 94720
}

C. C. MOORE

University of California

Berkeley, CA 94720

H. SAMELSON

Stanford University

Stanford, CA 94305

HAROLD STARK

University of California, San Diego La Jolla, CA 92093

\section{ASSOCIATE EDITORS}
R. ARENS
E. F. BECKENBACH (1906-1982)
B. H. NEUMANN
F. WOLF
K. YosHIDA

\section{SUPPORTING INSTITUTIONS}

\begin{abstract}
UNIVERSITY OF ARIZONA
UNIVERSITY OF BRITISH COLUMBIA

CALIFORNIA INSTITUTE OF TECHNOLOGY

UNIVERSITY OF CALIFORNIA

MONTANA STATE UNIVERSITY

UNIVERSITY OF NEVADA, RENO

NEW MEXICO STATE UNIVERSITY OREGON STATE UNIVERSITY
\end{abstract}

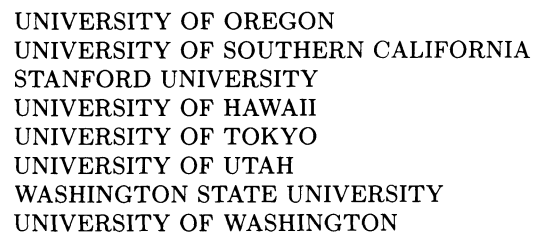

The Supporting Institutions listed above contribute to the cost of publication of this Journal, but they are not owners or publishers and have no responsibility for its content or policies.

Mathematical papers intended for publication in the Pacific Journal of Mathematics should be in typed form or offset-reproduced (not dittoed), double spaced with large margins. Please do not use built up fractions in the text of the manuscript. However, you may use them in the displayed equations. Underline Greek letters in red, German in green, and script in blue. The first paragraph must be capable of being used separately as a synopsis of the entire paper. In particular it should contain no bibliographic references. Please propose a heading for the odd numbered pages of less than 35 characters. Manuscripts, in triplicate, may be sent to any one of the editors. Please classify according to the scheme of Math. Reviews, Index to Vol. 39. Supply name and address of author to whom proofs should be sent. All other communications should be addressed to the managing editor, or Elaine Barth, University of California, Los Angeles, California 90024.

There are page-charges associated with articles appearing in the Pacific Journal of Mathematics. These charges are expected to be paid by the author's University, Government Agency or Company. If the author or authors do not have access to such Institutional support these charges are waived. Single authors will receive 50 free reprints; joint authors will receive a total of 100 free reprints. Additional copies may be obtained at cost in multiples of 50 .

The Pacific Journal of Mathematics is issued monthly as of January 1966. Regular subscription rate: $\$ 190.00$ a year (5 Vols., 10 issues). Special rate: $\$ 95.00$ a year to individual members of supporting institutions.

Subscriptions, orders for numbers issued in the last three calendar years, and changes of address should be sent to Pacific Journal of Mathematics, P.O. Box 969, Carmel Valley, CA 93924, U.S.A. Old back numbers obtainable from Kraus Periodicals Co., Route 100, Millwood, NY 10546.

The Pacific Journal of Mathematics at P.O. Box 969, Carmel Valley, CA 93924 (ISSN 0030-8730) publishes 5 volumes per year. Application to mail at Second-class postage rates is pending at Carmel Valley, California, and additional mailing offices. Postmaster: send address changes to Pacific Journal of Mathematics, P.O. Box 969, Carmel Valley, CA 93924.

PUBLISHED BY PACIFIC JOURNAL OF MATHEMATICS, A NON-PROFIT CORPORATION Copyright (C) 1986 by Pacific Journal of Mathematics 


\section{Pacific Journal of Mathematics}

Vol. 124, No. $2 \quad$ June, 1986

Philip Lee Bowers, Nonshrinkable "cell-like" decompositions of $s \ldots \ldots .257$

Aurelio Carboni and Ross Street, Order ideals in categories .......... 275

Leoni Dalla, Increasing paths on the one-skeleton of a convex compact set in

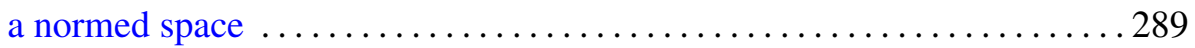

Jim Hoste, A polynomial invariant of knots and links ............... 295

Sheldon Katz, Tangents to a multiple plane curve ................... 321

Thomas George Lucas, Some results on Prüfer rings $\ldots \ldots \ldots \ldots \ldots \ldots 33$

Pham Anh Minh, Modular invariant theory and cohomology algebras of

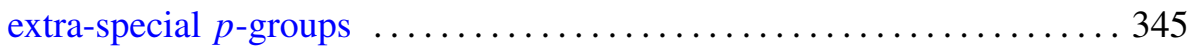

Ikuko Miyamoto, On inclusion relations for absolute Nörlund

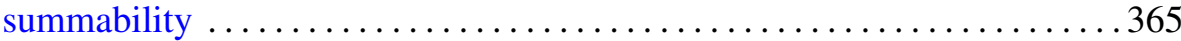

A. Papadopoulos, Geometric intersection functions and Hamiltonian flows on the space of measured foliations on a surface ............. 375

Richard Dean Resco, J. Toby Stafford and Robert Breckenridge

Warfield, Jr., Fully bounded $G$-rings $\ldots \ldots \ldots \ldots \ldots \ldots \ldots \ldots \ldots 4$

Haskell Paul Rosenthal, Functional Hilbertian sums .................417

Luen-Fai Tam, Regularity of capillary surfaces over domains with corners: borderline case ................................. 469

Hugh C. Williams, The spacing of the minima in certain cubic lattices .....483 